\title{
An Adaptive Importance Sampling Algorithm for Bayesian Inversion with Multimodal distributions
}

\author{
Weixuan $\operatorname{Li}^{\text {a }}$, Guang Lin ${ }^{\text {b } 1}$ \\ a Pacific Northwest National Laboratory, Richland, WA 99352, USA, \\ ${ }^{\mathrm{b}}$ Department of Mathematics and School of Mechanical Engineering, Purdue \\ University, West Lafayette, IN 47907, USA.
}

\begin{abstract}
Parametric uncertainties are encountered in the simulations of many physical systems, and may be reduced by an inverse modeling procedure that calibrates the simulation results to observations on the real system being simulated. Following Bayes' rule, a general approach for inverse modeling problems is to sample from the posterior distribution of the uncertain model parameters given the observations. However, the large number of repetitive forward simulations required in the sampling process could pose a prohibitive computational burden. This difficulty is particularly challenging when the posterior is multimodal. We present in this paper an adaptive importance sampling algorithm to tackle these challenges. Two essential ingredients of the algorithm are: 1) a Gaussian mixture (GM) model adaptively constructed as the proposal distribution to approximate the possibly multimodal target posterior, and 2) a mixture of polynomial chaos (PC) expansions, built according to the GM proposal, as a surrogate model to alleviate the computational burden caused by computational-demanding forward model evaluations. In three illustrative examples, the proposed adaptive importance sampling algorithm demonstrates its capabilities of automatically finding a GM proposal with an appropriate number of modes for the specific problem under study, and obtaining a sample accurately and efficiently representing the posterior with limited number of forward simulations.
\end{abstract}

Key words: inverse modeling, uncertainty reduction, adaptive sampling, Gaussian mixture, mixture of polynomial chaos expansions

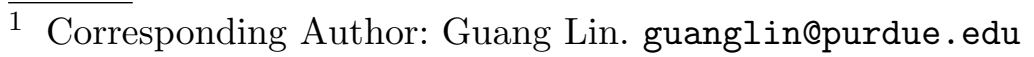




\section{Introduction}

Accurate simulations of physical systems using numerical models rely on model parameter values that correctly characterize the system being modeled. However, in many applications, the model parameters are subject to uncertainties, due to the lack of prior knowledge and the difficulty in direct measurement of the parameters. One path to parametric uncertainty reduction is to calibrate the simulation results to available observations on the corresponding quantities of the real system, which poses an inverse modeling problem, i.e., estimation of model input (parameters) from model output (simulation results).

Inverse problems usually have non-unique solutions, i.e., there exist different combinations of parameter values that all lead to simulation results matching observations. In such a situation, a regularization criterion may be used to identify a most favored solution. However, there is no guarantee that this solution reflects reality. In fact non-unique solutions indicate that the uncertainty of model parameters cannot be eliminated by the observations. A better treatment for such ill-posed inverse problems is to formulate them within the Bayesian framework, in which we compute the posterior distribution that represents the multiple solutions and quantifies the remaining parametric uncertainties $[1,2]$.

By Bayes' rule the posterior distribution is proportional to the product of the prior distribution, which represents the original parametric uncertainty, and the likelihood function, which conveys the information gained from the observations. But in practice, the posterior cannot be analytically derived except for a few simple situations. Instead, the posterior can only be approximated with some numerical approaches. A straightforward approach is to draw a sample of points from the posterior distribution, using a sampling algorithm such as importance sampling or Markov chain Monte Carlo (MCMC). Such algorithms involve two steps to obtain the desired sample points: proposal and correction. In the proposal step, parameter points are randomly generated from a proposal distribution, which may be biased from the target posterior but is easy to sample from. The proposal points are then adjusted in the correction step to represent the target posterior distribution.

One difficulty commonly encountered in the implementation of sampling algorithms is the selection of an appropriate proposal distribution. A common choice for the proposal is the prior distribution. However, in many Bayesian inversion problems, the posterior distribution concentrates only on a small fraction of the region covered by the prior distribution. As a result, most of the proposed points except a few would miss the target, and hence be eliminated from the final sample. This phenomenon, known as sample degeneracy, results in a poor representation of the posterior. Ideally, we prefer a proposal 
distribution that is close to the target. But this is not trivial because the target distribution itself is unknown in the context of a Bayesian inversion problem. One means to deal with this issue is to construct the proposal such that it gradually approaches the target posterior in an adaptive fashion $[3,4,5]$.

In the correction step, the posterior distribution needs to be evaluated (up to a constant proportionality) at the proposed points, which usually requires a forward model simulation at each point. This poses another major difficulty when the forward model is computationally expensive to solve, which is often the case for real applications. The computational cost for repetitive model simulations could be prohibitively high for the sampling algorithm to be implemented. A straightforward idea to reduce the computational cost in the sampling process is to replace the original forward model with a surrogate, i.e., a computationally inexpensive approximation of the input-output mapping governed by the original forward model. Examples of surrogate models used in solving inverse problems include Gaussian process [6,7], radial basis functions interpolation [8], and neural networks [9]. Among different types of surrogate models, the stochastic spectral representation, known as polynomial chaos (PC) expansion [10], has been shown to be very efficient in both forward uncertainty propagation $[11,12,13]$ and inverse modeling problems $[14,15]$. Furthermore, a PC surrogate could be adaptively built for a specific inverse problem so that the necessary approximation accuracy is achieved with relatively small computational effort [16,5]. Generally, the accuracy of a surrogate may be adaptively improved either globally (e.g., increasing the order of PC expansion) or locally (e.g., refinement of the surrogate adaptively at some important regions) in the parameter space. The latter is preferred in the context of sampling for Bayesian inversion, because what we mainly care about is only the surrogate accuracy within or near the regions of the proposal distribution, where the sample points are generated.

In this paper, we study the adaptive construction of both the proposal distribution and the locally accurate PC surrogate, for efficient sampling from the posterior distribution. In a previous research work [5], a proposal distribution that is close to the posterior, as well as a PC surrogate with respect to this proposal, is found in a series of re-sampling iterations controlled by an annealing scheme. For simplicity, the proposal is selected from the family of uncorrelated Gaussian distribution, and the PC is correspondingly chosen to be Hermite polynomials. Following this work, we study the use of a more flexible, yet still simple enough, family of distributions, namely Gaussian mixture (GM) models [17], to be the proposal. The flexibility of GM models allows for close approximations to different posterior distributions, especially when they are multimodal. But on the other hand, there is not a standard form of PC associated with GM distributions. Moreover, it has been shown that building PC representation for multimodal distributions, such as GMs is difficult, usually requiring many high-order terms [18]. To avoid this difficulty, we adopt 
the idea of mixture of PCs, i.e., to build separate Hermite PCs for each of the Gaussian component in the GM model. This allows for an accurate surrogate built with low-order terms because 1) each component is unimodal and 2) the random perturbation of each component is much smaller than that of the total mixture. This method is similar to multi-element generalized PC [19]. We apply these techniques to facilitate an importance sampling algorithm, though they also can be used in combination with other sampling algorithms such as MCMC.

The structure of the paper is as follows: in Section 2, we state the problem of importance sampling for Bayesian inversion with a briefly review of relative concepts. In Section 3, we present the implementation procedures and the convergence analysis of our proposed adaptive importance sampling algorithm. Specifically, we discuss how to adaptively construct the GM proposal and its corresponding PC surrogate. The algorithm is demonstrated with 3 examples in Section 4, and finally, we conclude the paper in Section 5 with some further discussions.

\section{Importance Sampling for Bayesian Inversion}

\subsection{Bayesian Inversion}

We first review the basic concepts of Bayesian inversion. Consider a forward simulation model $\mathbf{d}=g(\mathbf{m})$, where $\mathbf{m} \in \mathbb{R}^{n_{m}}$ is a vector that contains all the input parameters, whose values are subject to some uncertainty. $\mathbf{d}$ is the vector of simulation output variables which may be compared with the observations of the corresponding real system being simulated. In many applications, $g(\cdot)$ is a nonlinear model and computationally expensive to evaluate.

Because the exact values of the input parameters are not known, $\mathbf{m}$ is expressed as a random vector characterized by a prior density function $p(\mathbf{m})$, which reflects our limited knowledge about the system being simulated. One way to reduce the uncertainty associated with $\mathbf{m}$ is to calibrate the simulation results to the observations $\mathbf{d}^{*}$ of the real system. This poses an inverse problem, which may be formulated with Bayes' theorem,

$$
p\left(\mathbf{m} \mid \mathbf{d}^{*}\right)=h \cdot L(g(\mathbf{m})) p(\mathbf{m}) .
$$

The possible values of $\mathbf{m}$ given the observations are described by the posterior distribution $p\left(\mathbf{m} \mid \mathbf{d}^{*}\right)$, which is equal to the product of the prior probability density function (pdf) $p(\mathbf{m})$, the likelihood function $L(g(\mathbf{m}))$, and the normalizing factor $h=\left[\int L(g(\mathbf{m})) p(\mathbf{m}) d \mathbf{m}\right]^{-1}$. While $h$ is constant with respect 
to $\mathbf{m}$, and $p(\mathbf{m})$ is given as known, the key in estimating the posterior is the computation of the likelihood $L$, which reflects the additional information gained from the observed data.

By definition, the likelihood is the pdf of the observation $\mathbf{d}^{*}$ given the condition that $\mathbf{m}$ are the true model parameters, $L(g(\mathbf{m}))=p\left(\mathbf{d}^{*} \mid \mathbf{m}\right)$. When the observation is free of error and the model is an exact representation of the physical mechanism under study, we have $\mathbf{d}^{*}=\mathbf{d}=g(\mathbf{m})$, which means the observation is an exact mapping from the model parameters. So the likelihood is given by

$$
L(g(\mathbf{m}))=p\left(\mathbf{d}^{*} \mid \mathbf{m}\right)=\delta\left(\mathbf{d}^{*}-g(\mathbf{m})\right),
$$

where $\delta(\cdot)$ is the Dirac-delta function. Eq. (2) poses a very strong constraint on the posterior because it implies that a parameter point should be kept out of the posterior unless it exactly matches the observation through the model. However, in practice, neither the observation nor the model is perfect. Assuming the observation differs from the simulation by a random error e: $\mathbf{d}^{*}=\mathbf{d}+\mathbf{e}=g(\mathbf{m})+\mathbf{e}$. Then the likelihood is given by

$$
L(g(\mathbf{m}))=p\left(\mathbf{d}^{*} \mid \mathbf{m}\right)=p_{\mathbf{e}}\left(\mathbf{d}^{*}-g(\mathbf{m})\right),
$$

where $p_{\mathbf{e}}(\cdot)$ is the pdf of error e. Note that, to evaluate $L$ at a parameter point $\mathbf{m}$, one model simulation of $g(\cdot)$ is needed.

Although Bayes' rule (Eq.(1)) provides the theoretical foundation for computing the posterior pdf, it cannot be analytically and explicitly derived except for a few special cases. A general approximate solution is to draw a sample from the posterior pdf using a sampling algorithm, such as importance sampling.

\subsection{Importance Sampling}

Importance sampling draws a sample from a target distribution, e.g., the posterior $p\left(\mathbf{m} \mid \mathbf{d}^{*}\right)$, in two steps: proposal and correction. In the first step, a sample of points, $\left\{\mathbf{m}_{q}^{(1)}, \mathbf{m}_{q}^{(2)}, \ldots, \mathbf{m}_{q}^{\left(n_{S}\right)}\right\}$, is generated from a proposal distribution $q(\mathbf{m})$, which satisfies that $q(\mathbf{m})>0$, for all $\mathbf{m}$ such that $p\left(\mathbf{m} \mid \mathbf{d}^{*}\right)>0 . q(\mathbf{m})$ may be different from the target distribution but takes some simple form that is easy to sample from. In the second step, we make this proposed sample reflect the pdf of $p\left(\mathbf{m} \mid \mathbf{d}^{*}\right)$ by assigning each sample point a weight

$$
w_{i}=\frac{v_{i}}{\sum_{j=1}^{n_{S}} v_{j}}, \quad i=1,2, \ldots, n_{S},
$$

where

$$
v_{j}=\frac{L\left(g\left(\mathbf{m}_{q}^{(j)}\right)\right) p\left(\mathbf{m}_{q}^{(j)}\right)}{q\left(\mathbf{m}_{q}^{(j)}\right)}, \quad j=1,2, \ldots, n_{S} .
$$


With the sample points and weights, the posterior pdf can be approximated by

$$
p\left(\mathbf{m} \mid \mathbf{d}^{*}\right) \approx \sum_{i=1}^{n_{S}} w_{i} \delta\left(\mathbf{m}-\mathbf{m}_{q}^{(i)}\right)
$$

If equal-weight sample points are preferred (in case they need to be fed to a post-processing algorithm that by default takes equal-weight sample), they can be obtained by re-sampling among the proposed points of $\left\{\mathbf{m}_{q}^{(1)}, \ldots, \mathbf{m}_{q}^{\left(n_{S}\right)}\right\}$ with probability of picking the point $\mathbf{m}_{q}^{(i)}$ being $w_{i}$. For example, we may use the method of systematic re-sampling: generate $n_{S}$ i.i.d. random numbers $u_{j}$ from the uniform distribution $U(0,1)$, and let $\mathbf{m}^{(j)}=\mathbf{m}_{q}^{(r)}$, such that $\sum_{l=1}^{r} w_{l} \geq$ $u_{j}$ and $\sum_{l=1}^{r-1} w_{l}<u_{j}$. Finally, $S_{i}=\left\{\mathbf{m}^{(1)}, \ldots, \mathbf{m}^{\left(n_{S}\right)}\right\}$ forms an equal-weight sample representing the posterior pdf:

$$
p\left(\mathbf{m} \mid \mathbf{d}^{*}\right) \approx \frac{1}{n_{S}} \sum_{j=1}^{n_{S}} \delta\left(\mathbf{m}-\mathbf{m}^{(j)}\right) .
$$

\section{Adaptive Importance Sampling Algorithm}

\subsection{Basic Idea}

As discussed in the introduction Section, two issues may arise in the process of importance sampling. The first one is sample degeneracy. When the proposal distribution is not close to the posterior, most proposed points would be off the target and result in nearly zero weights, and hence a poor representation of the posterior. Secondly, repetitive forward model evaluations are required at each proposed point, which could easily exhaust available computational resources for computational-demanding forward models. To address these challenges we propose the following adaptive importance sampling algorithm.

Two techniques, i.e., GM proposal and mixture of PC surrogates, are used to address the challenges of sample degeneracy and computationally expensive forward simulations. As discussed in Section 1, a proposal distribution chosen from GM models is not only easy to sample from, but also flexible enough to approximate non-Gaussian, particularly multimodal posteriors. As for the mixture of PC surrogates, it provides a computationally efficient approximation of the original forward model at the proposed parameter points and can be used instead in the sampling algorithm to greatly speed up the process.

To find a proper GM proposal and the corresponding PC surrogates, the idea of annealed importance sampling [20] is adopted. Starting from an initial sample of points generated from the prior, $S_{0}=\left\{\mathbf{m}_{0}^{(1)}, \mathbf{m}_{0}^{(2)}, \ldots, \mathbf{m}_{0}^{\left(n_{S}\right)}\right\}$, we loop over 
a series of re-sampling iterations controlled by an annealing scheme. In the $i$ th iteration, a sample of $n_{S}$ points are drawn from an adjusted posterior pdf,

$$
p\left(\mathbf{m} ; \tau_{i} \mid \mathbf{d}^{*}\right)=h_{\tau_{i}} \cdot L(g(\mathbf{m}))^{1 / \tau_{i}} p(\mathbf{m}),
$$

where $\infty=\tau_{0}>\tau_{1}>\ldots>\tau_{i} \geq 1$ are the temperature parameter. As $\tau_{i}$ drops from $\infty$ to 1, Eq. (8) gradually deforms from the prior to the posterior.

By careful selection of the temperatures, the adjusted distributions are served as a bridge connecting the prior and the posterior. Since the adjusted distributions in two consecutive iterations are similar to each other, the sample obtained in previous iteration provides a guidance on where to propose new sample points in the current iteration. Specifically, we choose the proposal pdf for the $i$ th iteration to be a GM model that closely fits the sample $S_{i-1}=\left\{\mathbf{m}_{i-1}^{(1)}, \mathbf{m}_{i-1}^{(2)}, \ldots, \mathbf{m}_{i-1}^{\left(n_{S}\right)}\right\}$. Once we have a GM proposal in the current iteration, a PC surrogate can be built accordingly to approximate the forward model at the proposed points.

The work flow of the proposed algorithm is illustrated in Fig. 1, and the implementation details are discussed in following subsections.

\subsection{Build Gaussian Mixture Proposals}

The first step in the $i$ th re-sampling iteration of our adaptive importance sampling algorithm is to build a GM proposal distribution, which by definition is the superposition of $K$ different multivariate Gaussian pdfs:

$$
q(\mathbf{m} ; \mathbf{v})=\sum_{k=1}^{K} \pi_{k} \mathcal{N}\left(\mathbf{m} \mid \boldsymbol{\mu}_{k}, \boldsymbol{\Sigma}_{k}\right)
$$

A GM model is characterized by the parameters $\mathbf{v}=\left\{K, \pi_{k}, \boldsymbol{\mu}_{k}, \boldsymbol{\Sigma}_{k}\right\}$, where $K$ is the number of Gaussian components; $\pi_{k}$, which sum to 1 , are the probabilities of $K$ Gaussian components; $\boldsymbol{\mu}_{k}$ and $\boldsymbol{\Sigma}_{k}$ are the mean vectors and covariance matrices of $K$ Gaussian components, respectively. By adjusting the parameters $\mathbf{v}$, a GM model can flexibly mimic a non-Gaussian, especially a multimodal pdf. A sample point from pdf (Eq. (9)) can be obtained in two steps: 1) randomly pick a component $k$ with probability $\pi_{k}$ from GM model; and 2) generate a sample point from the Gaussian distribution $\mathcal{N}\left(\mathbf{m} \mid \boldsymbol{\mu}_{k}, \boldsymbol{\Sigma}_{k}\right)$.

In the $i$ th re-sampling iteration, we need to find a GM proposal pdf (i.e., to determine the parameter vector $\mathbf{v}=\left\{K, \pi_{k}, \boldsymbol{\mu}_{k}, \boldsymbol{\Sigma}_{k}\right\}$ ), which closely fits the sample obtained in the previous iteration. K-means clustering [21], and expectation-maximization (EM) algorithm [22,23] are widely used classic methods for building GM models. However, in these commonly used algorithms, 
the number of components $K$ has to be specified by users. More sophisticated methods, such as the "L" method [24] and variational Bayes [25], have been proposed to automatically determine $K$, though they are usually more computationally intensive.

In our study, we adopt the idea of hierarchical clustering [26,27], which automatically partitions the sample points into $K$ clusters such that each cluster approximately represents a unimodal component, and meanwhile, remains relatively simple and fast. Starting with $K=1$, the sample points $S=$ $\left\{\mathbf{m}^{(1)}, \mathbf{m}^{(2)}, \ldots, \mathbf{m}^{\left(n_{S}\right)}\right\}$ are first partitioned into two clusters, $S^{(1)}$ and $S^{(2)}$, with $\mathrm{k}$-means method. Then a test is implemented to decide whether the partition is necessary. If yes, we accept the partition and increase $K$ by one. Repeat the "partition-test" procedure for each cluster until no more subdivision is needed or a preset maximum $K$ is reached. After determining $K$ by hierarchical clustering algorithm, other parameters characterizing the GM model are determined as follows: probability of the $k$ th component is $\pi_{k}=n_{S}^{(k)} / n_{S}$, where $n_{S}^{(k)}$ and $n_{S}$ are the number of points in the $k$ th cluster and in the entire sample, respectively. $\boldsymbol{\mu}_{k}$ and $\boldsymbol{\Sigma}_{k}$ are chosen to be the sample mean and covariance of the $k$ th cluster, respectively.

To complete the hierarchical clustering method, we need a criterion to determine whether the partition of the sample $S$ into two clusters $S^{(1)}$ and $S^{(2)}$ should be accepted. Consider the sum of squared error $J_{e}(1)$ of the undivided sample $S$, and the "within cluster" sum of squared error $J_{e}(2)$ of the two divided clusters $S^{(1)}$ and $S^{(2)}$ :

$$
\begin{gathered}
J_{e}(1)=\sum_{\mathbf{m} \in S}\|\mathbf{m}-\mathbf{C}\|^{2}, \\
J_{e}(2)=\sum_{i=1}^{2} \sum_{\mathbf{m} \in S^{(i)}}\left\|\mathbf{m}-\mathbf{C}^{(i)}\right\|^{2},
\end{gathered}
$$

where $\mathbf{C}$ and $\mathbf{C}^{(i)}$ are the centers of $S$ and $S^{(i)}$, respectively. If the two clusters are indeed well separated, the partition of the sample would result in a significant decrease in the sum of squared error. In this paper, we adopt the criterion from [26] that $S$ should be divided if $J_{e}(2) / J_{e}(1)$ is smaller than a threshold $\beta$.

Remark 1. Note that for multidimensional sample points, the three centers $\mathbf{C}, \mathbf{C}^{(1)}$, and $\mathbf{C}^{(2)}$ always lie on the same line in the $n_{m}$ dimensional parameter space, which we call the "separation direction". Also, the total sum of squared error can be decomposed into different components associated with each dimension,

$$
\sum_{\mathbf{m} \in S}\|\mathbf{m}-\mathbf{C}\|^{2}=\sum_{\mathbf{m} \in S}\left(m_{1}-C_{1}\right)^{2}+\ldots+\sum_{\mathbf{m} \in S}\left(m_{n_{m}}-C_{n_{m}}\right)^{2} .
$$


Without loss of generality, assume the first dimension is the separation direction (we can always rotate the coordinate system to make it true), then all the components in (12) except the first would not be affected by the separation (because the coordinates of the centers remain unchanged except for the separation dimension). To simplify the test, we only include the components associated with the separation direction when computing $J_{e}(1)$ and $J_{e}(2)$. Similar idea was used in [27], where multidimensional data are first projected to the separation dimension before the test.

Remark 2. A detailed discussion for setting the threshold is seen in the reference [26]. In the illustrative examples of this paper, we select $\beta=0.3$. If the sample points to be separated are from a two-component (with equal probability) GM distribution, $0.5\left[\mathcal{N}\left(m \mid \mu_{1}, \sigma^{2}\right)+\mathcal{N}\left(m \mid \mu_{2}, \sigma^{2}\right)\right]$, setting $\beta=0.3$ (approximately) implies that we would like to divide the sample into two clusters when the distance between the two modes $\left|\mu_{1}-\mu_{2}\right|>2.5 \sigma$. Choosing smaller/greater $\beta$ might result in less/more clusters, and hence less/more components in the GM proposal. Nevertheless, this should not lead to a notable difference in the final sampling result because the bias in the proposal distribution would be corrected by the importance sampling weights.

\subsection{Build Mixture of Polynomial Chaos Surrogates}

After the proposal distribution is determined, a surrogate model that approximately maps the proposed parameter points to their corresponding output values is needed to reduce the computational cost for computational-demanding forward model evaluation. Here we choose the approach of PC expansion, which is an efficient alternative to Monte Carlo method for uncertainty representation and propagation.

A PC expansion approximates a random variable, or vector $\mathbf{x}$ with a polynomial function with respect to a set of independent standard Gaussian random variables $\boldsymbol{\xi}=\left[\xi_{1}, \ldots, \xi_{n_{\xi}}\right]^{T}$ :

$$
\mathbf{x} \approx \sum_{i=0}^{n_{\psi}} \mathbf{c}_{i} \psi_{i}(\boldsymbol{\xi})
$$

where the PC basis functions $\psi_{i}(\boldsymbol{\xi})$ are the multivariate Hermite polynomials with respect to $\boldsymbol{\xi}$, and $\mathbf{c}_{i}$ are the deterministic coefficients that control the distribution of $\mathbf{x}$. For instance, the 2nd-order Hermite PC expansion includes the following terms:

$$
\mathbf{x} \approx \mathbf{c}_{0}+\sum_{j=1}^{n_{\xi}} \mathbf{c}_{j} \xi_{j}+\sum_{j=1}^{n_{\xi}} \mathbf{c}_{j j} \frac{\left(\xi_{j}^{2}-1\right)}{\sqrt{2}}+\sum_{j=2}^{n_{\xi}} \sum_{k=1}^{j-1} \mathbf{c}_{j k} \xi_{j} \xi_{k}
$$


We now show that how PC expansion can be used as a surrogate to speed up the importance sampling process in our proposed algorithm. First, express input parameters $\mathbf{m}$ with a PC expansion such that $\mathbf{m}$ follows the previously determined GM proposal pdf. For simplicity, we first consider the case of $K=$ $1, q(\mathbf{m})=\mathcal{N}(\mathbf{m} \mid \boldsymbol{\mu}, \boldsymbol{\Sigma})$. In this situation, we have the eigen-decomposition:

$$
\mathbf{m}(\boldsymbol{\xi})=\boldsymbol{\mu}+\sum_{i=1}^{n_{m}} \sqrt{\lambda_{i}} \boldsymbol{\eta}_{i} \xi_{i}
$$

in which $\lambda_{i}$ and $\boldsymbol{\eta}_{i}$ are the $i$ th eigenvalue and eigenvector of the covariance matrix $\Sigma$, respectively. It is easy to verify that Eq. (15) gives the desired Gaussian proposal $q(\mathbf{m})$. Comparing with Eq. (14), we see that Eq. (15) can be seen as a first-order PC expansion with $n_{\xi}=n_{m}, \mathbf{c}_{0}=\boldsymbol{\mu}, \mathbf{c}_{i}=\sqrt{\lambda_{i}} \boldsymbol{\eta}_{i}$, and all higher-order coefficients being zeros.

Since the output $\mathbf{d}$ depends on $\mathbf{m}$ and hence is also a function of $\boldsymbol{\xi}$, it can be expressed with the same set of Gaussian random variables and the PC basis functions

$$
\mathbf{d}(\boldsymbol{\xi}) \approx \tilde{g}(\boldsymbol{\xi})=\sum_{i=0}^{n_{\psi}} \mathbf{c}_{i}^{\mathbf{d}} \psi_{i}(\boldsymbol{\xi}) .
$$

Note that the basis functions are orthogonal and normalized, $\left\langle\psi_{i} \psi_{j}\right\rangle=\delta_{i j}$, where $<>$ denotes the mathematical expectation, and $\delta_{i j}$ is the Kronecker delta. So the coefficients can be determined by projections

$$
\mathbf{c}_{i}^{\mathbf{d}}=<\mathbf{d}(\boldsymbol{\xi}) \psi_{i}(\boldsymbol{\xi})>, \quad i=0, \ldots, n_{\psi}
$$

In stochastic collocation method [29], Eq. (17) can be approximately solved with a numerical quadrature scheme

$$
\mathbf{c}_{i}^{\mathbf{d}} \approx \sum_{j=1}^{Q} \mathbf{d}\left(\boldsymbol{\xi}^{(j)}\right) \psi_{i}\left(\boldsymbol{\xi}^{(j)}\right) \gamma^{(j)}, \quad i=0, \ldots, n_{\psi},
$$

where $\boldsymbol{\xi}^{(j)}$ and $\gamma^{(j)}$ are the quadrature nodes and weights, respectively, and $\mathbf{d}\left(\boldsymbol{\xi}^{(j)}\right)=g\left(\mathbf{m}\left(\boldsymbol{\xi}^{(j)}\right)\right)$, which is computed by evaluating the forward model $g(\cdot)$ at the $Q$ nodes.

PC expansions for the model input in Eq. (15) and output in Eq. (16) serve as a surrogate to original model $g(\cdot)$. To propose a parameter point $\mathbf{m}^{(i)}$ and to approximately compute its corresponding output value $\tilde{g}^{(i)}$, a realization of the independent standard Gaussian vector $\boldsymbol{\xi}^{(i)}$ is needed to generate, which can be substituted into Eqs. (15) and (16), respectively.

Now let's consider the general situation in which the GM proposal contains multiple Gaussian components, $K>1$. It has been demonstrated that building $\mathrm{PC}$ expansions for multimodal pdfs is difficult, usually requiring many high order terms in the expansion to achieve needed accuracy[18]. To avoid this 
difficulty, instead of building one PC surrogate for the entire GM proposal, we construct $K$ separate PC surrogates for different Gaussian components. For each Gaussian component, we build PC expansions for input parameter $\mathbf{m}$ and output d like Eq.(15) and Eq.(16), respectively. Because of the separation, the random perturbation in each component is much smaller than that of the entire GM distribution. As a result, the needed approximation accuracy may be achieved with relatively low-order PC expansions. Fig. 2 illustrates an example of building PC surrogates for a two-component GM proposal distribution. It is seen that building separate PC surrogates for different components results in much better approximation accuracy at the proposed points than a single same-order PC surrogate for the entire GM distribution. In the following illustrative examples, for each Gaussian component, we choose the 2nd-order PC expansion without crossing terms, which contains $n_{\psi}=2 n_{\xi}+1$ terms. Correspondingly, the first level sparse grid quadrature based on Gauss-Hermite rule, which requires $Q=2 n_{\xi}+1$ nodes, is used to resolve the coefficients. The total number of original model evaluations required in this process is $Q K$.

Next, a surrogate for the entire GM proposal is formed from the mixture of the separate PC expansions,

$$
\begin{gathered}
\mathbf{m}(\boldsymbol{\xi}, \mathbf{z})=\prod_{k=1}^{K}\left(\mathbf{m}\left(\boldsymbol{\xi}, z_{k}=1\right)\right)^{z_{k}}=\prod_{k=1}^{K}\left(\boldsymbol{\mu}_{k}+\sum_{i=1}^{n_{m}} \sqrt{\lambda_{i}^{k}} \boldsymbol{\eta}_{i}^{k} \xi_{i}\right)^{z_{k}}, \\
\mathbf{d}(\boldsymbol{\xi}, \mathbf{z}) \approx \tilde{g}(\boldsymbol{\xi}, \mathbf{z})=\prod_{k=1}^{K}\left(\tilde{g}\left(\boldsymbol{\xi}, z_{k}=1\right)\right)^{z_{k}}=\prod_{k=1}^{K}\left(\sum_{i=0}^{n_{\psi}} \mathbf{c}_{i}^{\mathrm{d} k} \psi_{i}(\boldsymbol{\xi})\right)^{z_{k}} .
\end{gathered}
$$

Here $\mathbf{z}=\left\{z_{1}, \ldots, z_{K}\right\}$ is a $K$-dimensional binary random vector $\mathbf{z}=\left\{z_{1}, \ldots, z_{K}\right\}$ that satisfies $z_{k} \in\{0,1\}, \sum_{k=1}^{K} z_{k}=1$. This implies that any realization of $\mathbf{z}$ has only one element $z_{k}$ taking the value of 1 , while other elements have to be 0 . We use $z_{k}=1$ to indicate the event that the proposed point is generated from the $k$ th component and let the probability $\operatorname{Pr}\left(z_{k}=1\right)=\pi_{k}$. Note that conditional to $z_{k}=1$, Eq.(19) and Eq.(20) become Eq.(15) and Eq.(16) for the $k$ th component $\mathcal{N}\left(\mathbf{m} \mid \boldsymbol{\mu}_{k}, \boldsymbol{\Sigma}_{k}\right)$, respectively.

Similar to the single component $(K=1)$ case, Eq.(19) and Eq.(20) together provide a surrogate model to facilitate the importance sampling process. To propose a parameter point $\mathbf{m}^{(i)}$ and to approximately compute its corresponding output value $\tilde{g}^{(i)}$, we need to independently generate two points, $\boldsymbol{\xi}^{(i)}$ and $\mathbf{z}^{(i)}$, following their respective distributions, and substitute them into Eq.(19) and Eq.(20), respectively.

Also, similar to Eq.(4) and Eq.(5), the importance sampling weights are then approximated by

$$
\tilde{w}_{i}=\frac{\tilde{v}_{i}}{\sum_{j=1}^{n_{S}} \tilde{v}_{j}}, \quad i=1,2, \ldots, n_{S},
$$


where

$$
\tilde{v}_{j}=\frac{L\left(\tilde{g}^{(j)}\right)^{1 / \tau} p\left(\mathbf{m}_{q}^{(j)}\right)}{q\left(\mathbf{m}_{q}^{(j)}\right)}, \quad j=1,2, \ldots, n_{S}
$$

\subsection{Cooling Scheme}

As a final major part of the algorithm, a cooling scheme is set up (i.e., to determine the temperature in each iteration) that renders a smooth transition from the prior to the posterior via the intermediate adjusted distributions. In the $i$ th iteration, the proposed sample points according to their corresponding likelihood are sorted in descending order: $L\left(\tilde{g}^{(1)}\right)>L\left(\tilde{g}^{(2)}\right)>\ldots>L\left(\tilde{g}^{\left(n_{S}\right)}\right)$. To avoid the sample degeneracy, the temperature $\tau_{i}$ is selected such that at least the first $n_{\rho}$ points (e.g., let $n_{\rho}=0.15 n_{S}$ ) have an adjusted likelihood greater than or equal to a threshold, e.g., $10 \%$ of the largest likelihood value of any proposed point:

$$
L\left(\tilde{g}^{\left(n_{\rho}\right)}\right)^{1 / \tau_{i}} \geq 0.1 L\left(\tilde{g}^{(1)}\right)^{1 / \tau_{i}}
$$

which yields

$$
\tau_{i} \geq \frac{\ln \left[L\left(\tilde{g}^{(1)}\right) / L\left(\tilde{g}^{\left(n_{\rho}\right)}\right)\right]}{\ln 10} .
$$

Moreover, to ensure the temperature drops to 1 in finite iterations, a requirement that $\tau_{i} \leq \alpha \tau_{i-1}$, where $\alpha \in(0,1)$, is imposed.

\subsection{Summary of the Adaptive Importance Sampling Algorithm}

After discussing the implementation details of each step in previous subsections, we summarize the adaptive importance sampling algorithm here:

Algorithm 1: Adaptive importance sampling using PC surrogates and GM proposal

Initialization: initial sample from prior $p(\mathbf{m}): S_{0}=\left\{\mathbf{m}_{0}^{(1)}, \mathbf{m}_{0}^{(2)}, \ldots, \mathbf{m}_{0}^{\left(n_{S}\right)}\right\}$; $i=0 ; \tau_{0}=\infty$.

\section{Iteration:}

while $\tau_{i}>1$ do

1: $i \leftarrow i+1$

2: Fit a GM model $q\left(\mathbf{m} ; K, \pi_{k}, \boldsymbol{\mu}_{k}, \boldsymbol{\Sigma}_{k}\right)$ from sample points $S_{i-1}$.

3: Express model parameter $\mathbf{m}$ with Eq. (19) such that it follows the GM distribution $q\left(\mathbf{m} ; K, \pi_{k}, \boldsymbol{\mu}_{k}, \boldsymbol{\Sigma}_{k}\right)$.

4: Formulate PC representations of output $\mathbf{d}$ for each Gaussian component in the proposal using the stochastic collocation method, and then 
combine them as Eq. (20).

5: Propose a new sample of parameter points $S_{q}=\left\{\mathbf{m}_{q}^{(1)}, \mathbf{m}_{q}^{(2)}, \ldots, \mathbf{m}_{q}^{\left(n_{S}\right)}\right\}$ using Eq. (19).

6: Calculate approximate output $\tilde{g}^{(j)}$ and the corresponding likelihood values $L\left(\tilde{g}^{(j)}\right)$ for each proposed point using the PC surrogate (Eq. (20)).

7: Choose $\tau_{i}$ according to Eq. (24), and set the temperature $\tau_{i} \leftarrow \max \left(1, \min \left(\tau, \alpha \tau_{i-1}\right)\right)$.

8: Compute the weights $\tilde{w}_{j}$ using Eq. (21) and Eq. (22) with temperature $\tau_{i}$

9: Re-sample from $S_{q}$ with probability that $\mathbf{m}_{q}^{(j)}$ is drawn equal to $\tilde{w}_{j}$. This yields an equally weighted sample $S_{i}$. end while

\subsection{Convergence Analysis}

Here we show that the importance sampling results obtained with the mixture of PC surrogates converge in probability to that obtained with the true model following some simple assumptions.

Assumption 1. The PC surrogate for each component $\tilde{g}_{n_{\psi}, Q}\left(\boldsymbol{\xi}, z_{k}=1\right)$ converges to the original model $g\left(\boldsymbol{\xi}, z_{k}=1\right)$ in probability, for each $k$, as $n_{\psi}, Q \rightarrow \infty$.

Assumption 2. Likelihood $L(g(\mathbf{m}))$ is a continuous function with respect to model output $g(\mathbf{m})$.

The first assumption follows the well-known mean-square convergence property of PC approximations for $L_{2}$ random variables [11], which also implies convergence in probability. The second assumption is true when the error $\mathbf{e}$ between observation and simulated output follows a continuous pdf $p_{\mathbf{e}}$, e.g., a Gaussian distribution. Also, we recall two lemmas:

Lemma 3 (continuous mapping theorem). Suppose a sequence of random variables $X_{n} \rightarrow X$ in probability, and function $f(\cdot)$ has the set of discontinuity points $D_{f}$ such that $\operatorname{Pr}\left[X \in D_{f}\right]=0$. Then $f\left(X_{n}\right) \rightarrow f(X)$ in probability.

Lemma 4. If two sequences of random variables $X_{n} \rightarrow X, Y_{n} \rightarrow Y$ both in probability, then $X_{n} \pm Y_{n} \rightarrow X \pm Y, X_{n} Y_{n} \rightarrow X Y$, all in probability [30].

With the support of above assumptions and lemmas, we have the following Theorem 5. The results hold for each annealed re-sampling iteration in Algorithm 1, so we drop the temperature parameter $\tau$ in the following discussion.

Theorem 5. Sample from the posterior distribution $p\left(\mathbf{m} \mid \mathbf{d}^{*}\right)$ in a Bayesian inversion problem using Algorithm 1. $\mathbf{m}_{q}^{(i)}=\mathbf{m}\left(\boldsymbol{\xi}^{(i)}, \mathbf{z}^{(i)}\right), i=1, \ldots, n_{S}$ are a set 
of parameter points proposed from the GM proposal. $g^{(i)}=g\left(\boldsymbol{\xi}^{(i)}, \mathbf{z}^{(i)}\right)$ are the original model outputs at the proposed parameter points. $\tilde{g}^{(i)}=\tilde{g}_{n_{\psi}, Q}\left(\boldsymbol{\xi}^{(i)}, \mathbf{z}^{(i)}\right)$ are the approximate outputs given by the mixture of PC surrogates. $w_{i}$ are the importance sampling weights computed from $g^{(i)}$ with Eqs. (4) and (5). $\tilde{w}_{i}$ are the weights computed from $\tilde{g}^{(i)}$ with Eqs. (21) and (22). Suppose that

Assumptions 1 and 2 hold. Then we have $\tilde{w}_{i}$ converge in probability to $w_{i}$, $i=1, \ldots, n_{S}$, as $n_{\psi}, Q \rightarrow \infty$.

Proof. For any positive real number $\epsilon$, by law of total probability, we have

$$
\begin{aligned}
& \operatorname{Pr}\left(\left|g(\boldsymbol{\xi}, \mathbf{z})-\tilde{g}_{n_{\psi}, Q}(\boldsymbol{\xi}, \mathbf{z})\right|>\epsilon\right) \\
= & \sum_{k=1}^{K} \operatorname{Pr}\left(z_{k}=1\right) \operatorname{Pr}\left(\left|g\left(\boldsymbol{\xi}, z_{k}=1\right)-\tilde{g}_{n_{\psi}, Q}\left(\boldsymbol{\xi}, z_{k}=1\right)\right|>\epsilon\right) \\
= & \sum_{k=1}^{K} \pi_{k} \operatorname{Pr}\left(\left|g\left(\boldsymbol{\xi}, z_{k}=1\right)-\tilde{g}_{n_{\psi}, Q}\left(\boldsymbol{\xi}, z_{k}=1\right)\right|>\epsilon\right) .
\end{aligned}
$$

By Assumption 1, we have

$$
\lim _{n_{\psi}, Q \rightarrow \infty} \operatorname{Pr}\left(\left|g\left(\boldsymbol{\xi}, z_{k}=1\right)-\tilde{g}_{n_{\psi}, Q}\left(\boldsymbol{\xi}, z_{k}=1\right)\right|>\epsilon\right)=0, \quad k=1, \ldots, K .
$$

It follows from Eqs. (25) and (26) that

$$
\lim _{n_{\psi}, Q \rightarrow \infty} \operatorname{Pr}\left(\left|g(\boldsymbol{\xi}, \mathbf{z})-\tilde{g}_{n_{\psi}, Q}(\boldsymbol{\xi}, \mathbf{z})\right|>\epsilon\right)=0
$$

which means that $\tilde{g}^{(i)}$ converges to $g^{(i)}$ in probability, for $i=1, \ldots, n_{S}$. Next, by Assumption 2 and Lemma 3, we have

$$
L\left(\tilde{g}^{(i)}\right) \rightarrow L\left(g^{(i)}\right), \quad i=1, \ldots, n_{S}
$$

in probability, as $n_{\psi}, Q \rightarrow \infty$. Finally, by Lemma 3 and Lemma 4, we have

$$
\tilde{w}_{i} \rightarrow w_{i}, \quad i=1, \ldots, n_{S}
$$

in probability, as $n_{\psi}, Q \rightarrow \infty$.

\section{Illustrative Examples}

In this section, we demonstrate the accuracy and efficiency of the proposed adaptive importance sampling algorithm with 3 illustrative examples of different physical models. Each physical model includes some uncertain parameters that need to be estimated from some indirect observations. For each observation, we assume a normally distributed error with zero mean and a standard deviation equal to $5 \%$ of the observed value. 


\subsection{Example 1: Contamination Source Identification}

Consider the process of contaminant diffusion in a two-dimensional domain, which is governed by the (dimensionless) diffusion equation

$$
\frac{\partial u(\mathbf{x}, t)}{\partial t}=D \Delta u(\mathbf{x}, t), \quad \mathbf{x} \in \Omega=[-1,1]^{2}, \quad t \in[0, \infty),
$$

and is subject to boundary condition

$$
u(\mathbf{x}, t)=0, \quad \mathbf{x} \in \partial \Omega,
$$

where $u(\mathbf{x}, t)$ is the concentration at location $\mathbf{x}$ and time $t, D$ is the diffusion coefficient. At time $t=0$, a certain amount of contaminant is released into the domain at a location $\mathbf{x}_{0}$. Initial concentration distribution is approximated by

$$
u(\mathbf{x}, 0)=\frac{M}{2 \pi h^{2}} \exp \left(\frac{-\left|\mathbf{x}_{0}-\mathbf{x}\right|^{2}}{2 h^{2}}\right)
$$

where $M$ is the total amount of released contaminant, $h$ is the "radius" of the initially contaminated area. We assume $D=1$ and $h=0.025$ are known parameters, whereas the two coordinates of the contaminant source $\mathbf{x}_{0}$ and the released amount $M$ are three unknown parameters subject to uniform prior uncertainties: $\mathbf{x}_{0} \sim U\left([-1,1]^{2}\right), M \sim U([0,20])$.

Our objective is to infer the uncertain parameters from concentrations measurements. In particular, two case studies are considered, in which different measurements are available. In Case 1 , two sensors are located at $(-0.4,-0.4)$ and $(0,0.4)$. In Case 2 , one more sensor is located at $(0.4,-0.4)$, as illustrated in Fig. 3. In both case studies the sensors report the concentration measurements at two time snap shots $\left(t_{1}=0.04\right.$ and $\left.t_{2}=0.08\right)$.

Adaptive sampling processes in Case 1 and Case 2 are shown in Figs. 4 and 5 , respectively. The proposed points in the 1st iteration are generated from the prior pdf, whereas in the following iterations, the proposed points are generated from GM models fitted from the sample points obtained in previous iterations. For each Gaussian component, a PC surrogate is built locally to facilitate the sampling process. It is seen from the figures that our algorithm is capable of adaptively selecting different number of Gaussian components in different iterations as well as in different case studies. As temperature drops to 1 , samples from the posterior distributions are achieved. In case 1, a bimodal posterior pdf is finally achieved, which indicates that the concentration observations collected at the two sensors are not sufficient to determine the source location. In case 2 , where 3 sensors are employed, a unimodal posterior pdf is obtained, which reveals a significant reduction of the posterior uncertainty brought by the 3rd sensor. 
To verify the accuracy of our proposed algorithm, we also generate a sample from the posterior by running the original forward model. Since this diffusion problem is relatively inexpensive to simulate, we can afford a large number of simulations of the original model. This sample is viewed as the benchmark, because it does not incur a surrogate error. The contours of the 2-dimensional marginal pdfs computed from the two posterior samples (with original and surrogate models, respectively) of Case 1 are plotted in Fig. 6. As shown in Fig. 6, the two results are pretty close to each other. To further check the efficiency of our approach, the same problem is solved with 2 more different surrogate models, i.e., the level-2 and level-3 sparse grid interpolations based on Gauss-Patterson rule. Without adaptivity, these two surrogate models are directly built according to the prior distribution. The computational cost and the accuracy of the three surrogate-based sampling methods are shown in Fig. 7. The accuracy of each method is measured by the relative entropy of the estimated posterior compared to the benchmark. Fig. 7 indicates that our method is more accurate than the sparse grid surrogates with similar computational cost. Furthermore, the adaptive importance sampling method allows for a flexible allocation of computational resources. For example, it uses less Gaussian components, and hence less computational effort, for the unimodal case than the bimodal case.

\subsection{Example 2: Advection-Dispersion Model Calibration with Bimodal Prior}

To further test our algorithm, we apply it to a more complex advectiondispersion model previously studied in [31]. The 2-D model describes a conservative solute transport process in a confined aquifer as shown in Fig. 8. We assume the aquifer has homogeneous and isotropic conductivity $K$ and effective porosity $\phi$, and is bounded by two rivers with constant head at the left $\left(h_{1}=30[\mathrm{~L}]\right)$ and the right $\left(h_{2}=20[\mathrm{~L}]\right)$ boundaries, respectively, which results in a uniform background flow from left to right. An amount $Q_{0}$ of contaminant is accidentally released instantaneously into the aquifer at location $\left(x_{0}, y_{0}\right)$ and time $t_{0}$. The concentration of the solute $C$ can be solved from the advection-dispersion equation:

$$
\frac{\partial(\phi C)}{\partial t}=-\nabla \cdot(\phi C \mathbf{v}-\phi \mathbf{D} \nabla C)
$$

in which $\mathbf{D}$ is the dispersion tensor with the longitudinal and transverse components being $D_{L}=\alpha_{L}|\mathbf{v}|$ and $D_{T}=\alpha_{T}|\mathbf{v}|$, respectively. $\alpha_{L}$ and $\alpha_{T}$ are longitudinal and transverse dispersivities. The linear velocity of water flux $\mathbf{v}$ is determined by Darcy's law and continuity equation:

$$
\mathbf{v}=-\frac{1}{\phi} K \nabla h
$$




$$
\nabla \cdot(\phi \mathbf{v})=0
$$

where $h$ is the hydraulic head. The simulation of the advection-dispersion model is subject to 8 uncertain parameters with uniform prior densities distributed within the intervals listed in Table 2 . We assume that the aquifer is comprised of one of two possible types of media: type $A$ with high porosity and high conductivity $(\phi, K) \in[0.22,0.25] \times[24,30]$, or type $B$ with low porosity and low conductivity $(\phi, K) \in[0.15,0.18] \times[10,16]$. Such an assumption yields a bimodal prior. The concentration breakthrough curves are recorded at 6 observation wells located at the downstream of the contamination source, and are used to infer the uncertain model parameters.

We apply our adaptive importance sampling algorithm to this problem. From Table 1, it can be observed that the final sample from the posterior distribution is obtained in 5 iterations, which require a total number of 119 forward model evaluations. Initially, the algorithm chose 2 components in the GM proposal, which correctly reflects the bimodal prior. As the adaptive iteration progressed, the algorithm reduced the number of components to 1, indicating that the posterior uncertainty is reduced to a unimodal distribution with the information gained from the observations. The uncertainty reduction can be also seen from the histograms of the parameters (Figs. 9 and 10) plotted from the initial and final samples. The initial sample points follow the uniform prior distributions, and show the two possible types of media, whereas the final sample reveal a much narrower posterior distribution but still incorporates the reference (the synthetic true) parameter point. Furthermore, it is determined correctly that this aquifer is made of the type $B$ media of low porosity and low conductivity.

To validate the results, we show the simulated breakthrough curves from the initial and final samples, in comparison with the reference (Figs. 11 and 12). It can be observed that the simulations from the prior distribution reveal a great variability and do not correctly predict the observations. But the sample points from the posterior distribution lead to simulations that well match the observations.

\subsection{Example 3: History Matching of a Faulted Petroleum Reservoir}

In the third example, we apply our algorithm to the history matching problem of a petroleum reservoir model known as I-C Fault model [32,33]. We give a short introduction of the model, while more details could be found in the above cited references. The I-C Fault model represents the 2-D vertical cross section of an oil reservoir that is $1000 \mathrm{ft}$ wide and $60 \mathrm{ft}$ thick, and consists of six layers of alternating high- and low-permeable sand (Fig.13). The thickness of the six 
layers is an arithmetic progression from the bottom ( $7.5 \mathrm{ft})$ to the top $(12.5 \mathrm{ft})$, and a vertical fault in the middle divides the reservoir into two parts. Oil is recovered from the reservoir by water flooding, which utilizes an injection well injecting water into the reservoir at the left boundary, and a production well producing fluids at the right boundary. Both wells are completed on all layers and are operated at constant pressures. The upper and lower boundaries are bounded by no flow boundary conditions.

The model is characterized with three input parameters, which are associated with independent and uniform prior uncertainties: the fault throw (i.e., the vertical displacement at the fault) $h \in[0,60] \mathrm{ft}$, the permeability of the goodquality sand $k_{g} \in[100,200] \mathrm{md}$, and the permeability of the poor-quality sand $k_{p} \in[0,50]$ md. For each parameter point sampled from the prior distribution, the corresponding production history, i.e., the time series of oil production rate and water cut (the fraction of water in the total liquids produced), can be simulated from the model. Fig. 15 shows the simulated production histories based on a sample of input parameter points drawn from the prior pdf. We see that the variations of the input parameters cause a large uncertainty in the simulation results. To reduce the uncertainty, the input parameters are calibrated to the observations of the "synthetic true" production history, which is generated from the simulation based on a reference parameter point $\left(h_{0}=\right.$ $\left.10.4 \mathrm{ft}, k_{g 0}=131.6 \mathrm{md}, k_{p 0}=1.3 \mathrm{md}\right)$. Specifically, the observation data used for calibration are oil production rate and water cut recorded every month in the first three years of production.

The adaptive importance sampling algorithm obtained a sample of the posterior in 3 adaptive iterations, as shown in Table 1. From Fig. 14, we see that the algorithm started with one component for the GM proposal. 3 components are then detected in the following iterations, and hence a GM of 3 components is used to better approximate the multimodal posterior distribution. To validate the sample, we plot the simulated production history from the posterior sample in comparison with the synthetic true, as shown in Fig. 15. Again, we see a good match between the simulations and the observation. The total number of simulations of the original model required in this process is 49 . We see a great efficiency improvement comparing with a previous study on the same problem using genetic algorithms and artificial neural network approaches, where thousands of model simulations are needed [34].

\section{Concluding Discussions}

We present in this paper an adaptive importance sampling algorithm for Bayesian inverse modeling with multimodal distributions. To tackle the difficulty of sampling from a multimodal posterior and to enhance sampling 
efficiency, we use a GM model as the proposal distribution. The GM model is adaptively constructed to approximate different posterior distributions in different problems. Furthermore, we study the use of a mixture of PC expansions, built according to the GM proposal, as a surrogate model to alleviate the computational burden caused by forward model evaluations in the sampling process. Tested with three different inverse problems, the developed algorithm shows its capabilities of 1) finding the GM with a proper number of components as the proposal distribution that approximates the target posterior and thus enhance sampling efficiency, 2) building a mixture of locally accurate PC surrogates to avoid the expensive simulations of the original model required in the sampling process, and 3) obtaining a sample of points that well represent the multimodal posterior with limited simulations of the original forward model.

The majority of the computational cost in the algorithm is spent on the model evaluations at the collocation points, which are needed to build the PC surrogate. In our algorithm, we build separate PC expansion for each Gaussian component instead of a single PC expansion for the entire GM proposal. Seemingly, this method causes an extra computational burden since its computational cost is proportional to the number of Gaussian components. However, it in fact could be an economic choice because the separation of different Gaussian component avoids a large number of high-order terms in the PC expansion which would be necessary when approximating multimodal distributions.

PC based approaches are usually sensitive to the dimensionality (the number of input parameters) of the problem because the computational cost grows fast as the dimensionality increases. In our algorithm, the separation of the proposal into different Gaussian component allows for relatively accurate PC expansions with only low-order terms and a low-level collocation scheme, which mitigates the curse of dimensionality to some extent. Nevertheless, characterizing a very high-dimensional pdf, especially a multimodal one, with a sample of points is overwhelmingly difficult due to the extreme sparsity of high-dimensional space. As a rule of thumb, we suggest the algorithm being applied to problems with less than 10 20 parameters, depending on how many forward simulations the user can afford. For higher-dimensional problems, it is better to first re-parameterize the problem such that it is represented with fewer parameters (dimension reduction), rather than to directly sampling from the high-dimensional pdf. Our current research is going toward this direction.

Another direction of future work is to implement the algorithm within a sequential importance sampling framework for sequential data assimilation problems.

Acknowledgements: This work is supported by the U.S. Department of 
Energy, Office of Science, Office of Advanced Scientific Computing Research, Applied Mathematics program as part of the Multifaceted Mathematics for Complex Energy Systems ( $\mathrm{M}^{2} \mathrm{ACS}$ ) project and part of the Collaboratory on Mathematics for Mesoscopic Modeling of Materials project. This work is also partially supported by NSF Grant DMS-1115887. Computations were performed using the computational resources of Pacific Northwest National Laboratory (PNNL) Institutional Computing cluster systems and the National Energy Research Scientific Computing Center at Lawrence Berkeley National Laboratory. The PNNL is operated by Battelle for the US Department of Energy under Contract DE-AC05-76RL01830.

\section{References}

[1] J. Kaipio, E. Somersalo, Statistical and computational inverse problems, Vol. 160, Springer, 2005.

[2] A. M. Stuart, Inverse problems: a bayesian perspective, Acta Numerica 19 (2010) 451-559.

[3] J. M. Keith, D. P. Kroese, G. Y. Sofronov, Adaptive independence samplers, Statistics and Computing 18 (4) (2008) 409-420.

[4] O. Capp, R. Douc, A. Guillin, J.-M. Marin, C. P. Robert, Adaptive importance sampling in general mixture classes, Statistics and Computing 18 (4) (2008) 447-459. doi:10.1007/s11222-008-9059-x.

[5] J. Li, Y. M. Marzouk, Adaptive construction of surrogates for the bayesian solution of inverse problems, SIAM Journal on Scientific Computing 36 (2014) A1163-A1186.

[6] M. C. Kennedy, A. O'Hagan, Bayesian calibration of computer models, Journal of the Royal Statistical Society: Series B (Statistical Methodology) 63 (3) (2001) 425-464.

[7] D. Higdon, M. Kennedy, J. C. Cavendish, J. A. Cafeo, R. D. Ryne, Combining field data and computer simulations for calibration and prediction, SIAM Journal on Scientific Computing 26 (2) (2004) 448-466. doi:10.1137/ S1064827503426693.

[8] N. Bliznyuk, D. Ruppert, C. Shoemaker, R. Regis, S. Wild, P. Mugunthan, Bayesian calibration and uncertainty analysis for computationally expensive models using optimization and radial basis function approximation, Journal of Computational and Graphical Statistics 17 (2) (2008) 270-294. doi:10.1198/ $106186008 \times 320681$.

[9] R. Zou, W.-S. Lung, J. Wu, Multiple-pattern parameter identification and uncertainty analysis approach for water quality modeling, Ecological Modelling 220 (5) (2009) 621-629. doi:10.1016/j.ecolmodel.2008.11.021. 
[10] N. Wiener, The homogeneous chaos, American Journal of Mathematics 60 (4) (1938) 897-936.

[11] R. G. Ghanem, P. D. Spanos, Stochastic Finite Elements: A Spectral Approach, Courier Dover Publications, 2003.

[12] D. Xiu, G. E. Karniadakis, Modeling uncertainty in flow simulations via generalized polynomial chaos, Journal of Computational Physics 187 (1) (2003) $137-167$.

[13] W. Li, Z. Lu, D. Zhang, Stochastic analysis of unsaturated flow with probabilistic collocation method, Water Resources Research 45 (8) (2009) W08425 1-13.

[14] G. Saad, R. Ghanem, Characterization of reservoir simulation models using a polynomial chaos-based ensemble kalman filter, Water Resources Research 45 (4) (2009) W04417 1-19.

[15] Y. M. Marzouk, H. N. Najm, L. A. Rahn, Stochastic spectral methods for efficient bayesian solution of inverse problems, Journal of Computational Physics 224 (2) (2007) 560-586. doi:10.1016/j.jcp.2006.10.010.

[16] W. Li, G. Lin, D. Zhang, An adaptive ANOVA-based PCKF for highdimensional nonlinear inverse modeling, Journal of Computational Physics 258 (2014) 752-772. doi:10.1016/j.jcp.2013.11.019.

[17] G. McLachlan, D. Peel, Finite Mixture Models, John Wiley \& Sons, 2004.

[18] A. Nouy, Identification of multi-modal random variables through mixtures of polynomial chaos expansions, Comptes Rendus Mcanique 338 (12) (2010) 698703. doi:10.1016/j.crme.2010.09.003.

[19] X. Wan, G. E. Karniadakis, An adaptive multi-element generalized polynomial chaos method for stochastic differential equations, Journal of Computational Physics 209 (2) (2005) 617-642. doi:10.1016/j.jcp.2005.03.023.

[20] R. M. Neal, Annealed importance sampling, Statistics and Computing 11 (2) (2001) 125-139. doi:10.1023/A:1008923215028.

[21] J. A. Hartigan, M. A. Wong, Algorithm AS 136: A k-means clustering algorithm, Journal of the Royal Statistical Society. Series C (Applied Statistics) 28 (1) (1979) 100-108.

[22] A. P. Dempster, N. M. Laird, D. B. Rubin, Maximum likelihood from incomplete data via the EM algorithm, Journal of the Royal statistical Society 39 (1) (1977) $1-38$.

[23] C. J. Wu, On the convergence properties of the EM algorithm, The Annals of statistics (1983) 95-103.

[24] S. Salvador, P. Chan, Determining the number of clusters/segments in hierarchical clustering/segmentation algorithms, in: 16th IEEE International Conference on Tools with Artificial Intelligence, 2004. ICTAI 2004, 2004, pp. 576-584. doi:10.1109/ICTAI.2004.50. 
[25] C. M. Bishop, Pattern recognition and machine learning, springer New York, 2006.

[26] R. O. Duda, P. E. Hart, D. G. Stork, Pattern Classification, John Wiley \& Sons, 2001.

[27] G. Hamerly, C. Elkan, Learning the k in k-means, in: NIPS, Vol. 3, 2003, pp. $281-288$.

[28] C. Ding, X. He, H. Zha, H. Simon, Adaptive dimension reduction for clustering high dimensional data, in: 2002 IEEE International Conference on Data Mining, 2002. ICDM 2003. Proceedings, 2002, pp. 147-154. doi:10.1109/ICDM. 2002. 1183897.

[29] D. Xiu, Efficient collocational approach for parametric uncertainty analysis, Communications in computational physics 2 (2) (2007) 293-309.

[30] K. L. Chung, A Course in Probability Theory, Academic Press, 2001.

[31] W. Li, Inverse modeling and uncertainty quantification of nonlinear flow in porous media models, Ph.D. thesis, University of Southern California (2014).

[32] Z. Tavassoli, J. Carter, P. King, Errors in history matching, SPE Journal 9 (3) (2004) 352-361. doi:10.2118/86883-PA.

[33] J. Carter, P. Ballester, Z. Tavassoli, P. King, Our calibrated model has poor predictive value: An example from the petroleum industry, Reliability Engineering \& System Safety 91 (1011) (2006) 1373-1381. doi:10.1016/j . ress.2005.11.033.

[34] M. Christie, V. Demyanov, D. Erbas, Uncertainty quantification for porous media flows, Journal of Computational Physics 217 (1) (2006) 143-158. doi: $10.1016 / j \cdot j c p .2006 .01 .026$.

\section{Tables}

Table 1

Number of Gaussian components selected for the GM proposal in different iterations and in different examples.

\begin{tabular}{llllll}
\hline & Iteration 1 & Iteration 2 & Iteration 3 & Iteration 4 & Iteration 5 \\
\hline Case 1, Ex. 1 & 1 & 4 & 2 & 2 & - \\
Case 2, Ex. 1 & 1 & 2 & 1 & 1 & - \\
Ex. 2 & 2 & 2 & 1 & 1 & 1 \\
Ex. 3 & 1 & 3 & 3 & - & - \\
\hline
\end{tabular}


Table 2

List of uncertain parameters and corresponding uncertain ranges in Example 2.

\begin{tabular}{lllll}
\hline $\begin{array}{l}\text { Parameters } \\
\text { [unit] }\end{array}$ & $x_{0}[\mathrm{~L}]$ & $y_{0}[\mathrm{~L}]$ & $Q_{0}[\mathrm{M}]$ & $t_{0}[\mathrm{~T}]$ \\
Range & {$[50,150]$} & {$[-50,50]$} & {$[0.5,1.5] \times 10^{6}$} & {$[0,60]$} \\
\hline $\begin{array}{l}\text { Parameters } \\
{[\text { unit }]}\end{array}$ & $\phi$ & $K\left[\mathrm{LT}^{-1}\right]$ & $\alpha_{L}[\mathrm{~L}]$ & $\alpha_{T}[\mathrm{~L}]$ \\
Range & {$[0.15,0.18] \cup[0.22,0.25]$} & {$[10,16] \cup[24,30]$} & {$[1.5,2.5]$} & {$[0.6,1]$} \\
\hline
\end{tabular}

\section{Figures}

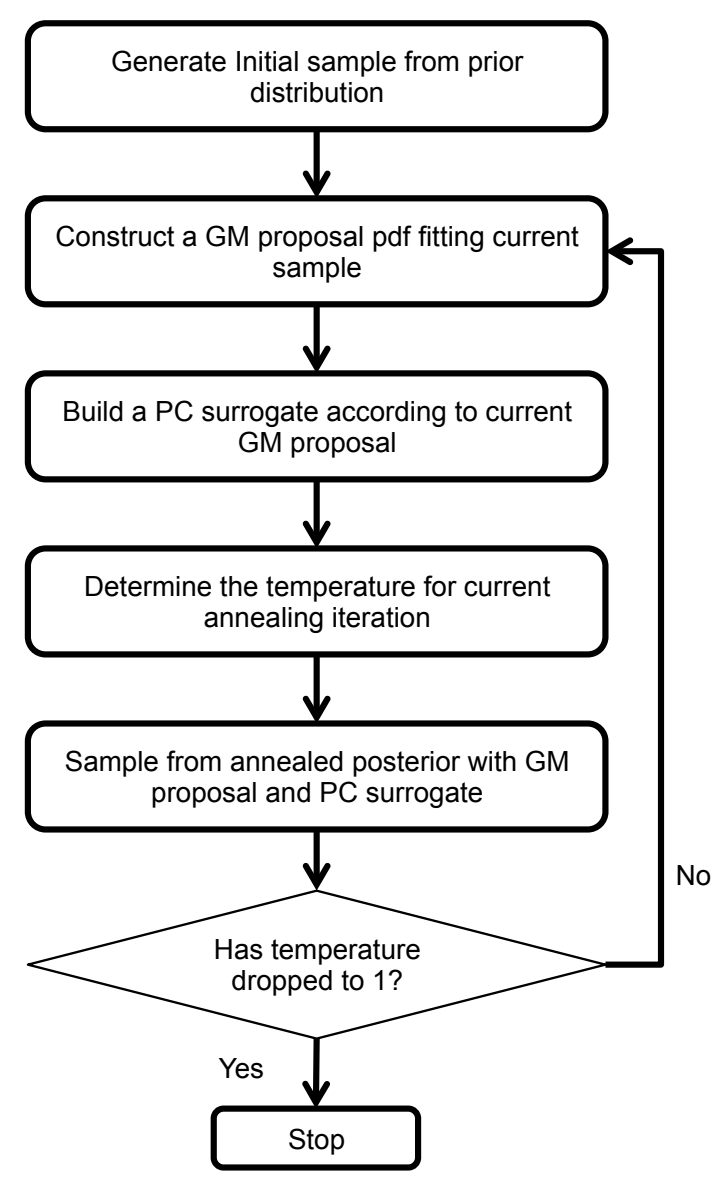

Fig. 1. Work flow of adaptive importance sampling with GM proposal and mixture of PC surrogates. 



Fig. 2. Two different ways of building PC surrogate for parameter points proposed from a Gaussian mixture distribution. Left: build a single PC surrogate for the Gaussian mixture proposal distribution. Right: build separate PC surrogates for each of the two Gaussian components and form a mixture of PC surrogates. 


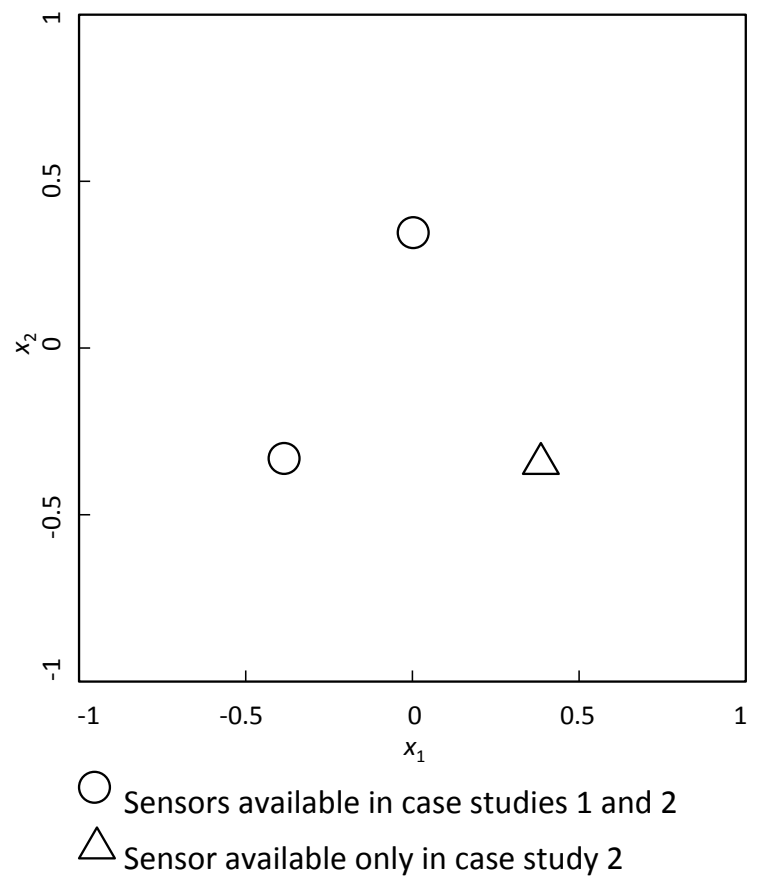

Fig. 3. Concentration sensors in the domain of contaminant diffusion (Problem 1). 


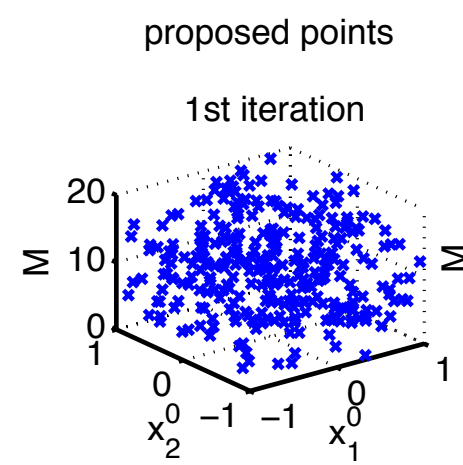

sampled points

marginal pdf of source locatoin

2nd iteration
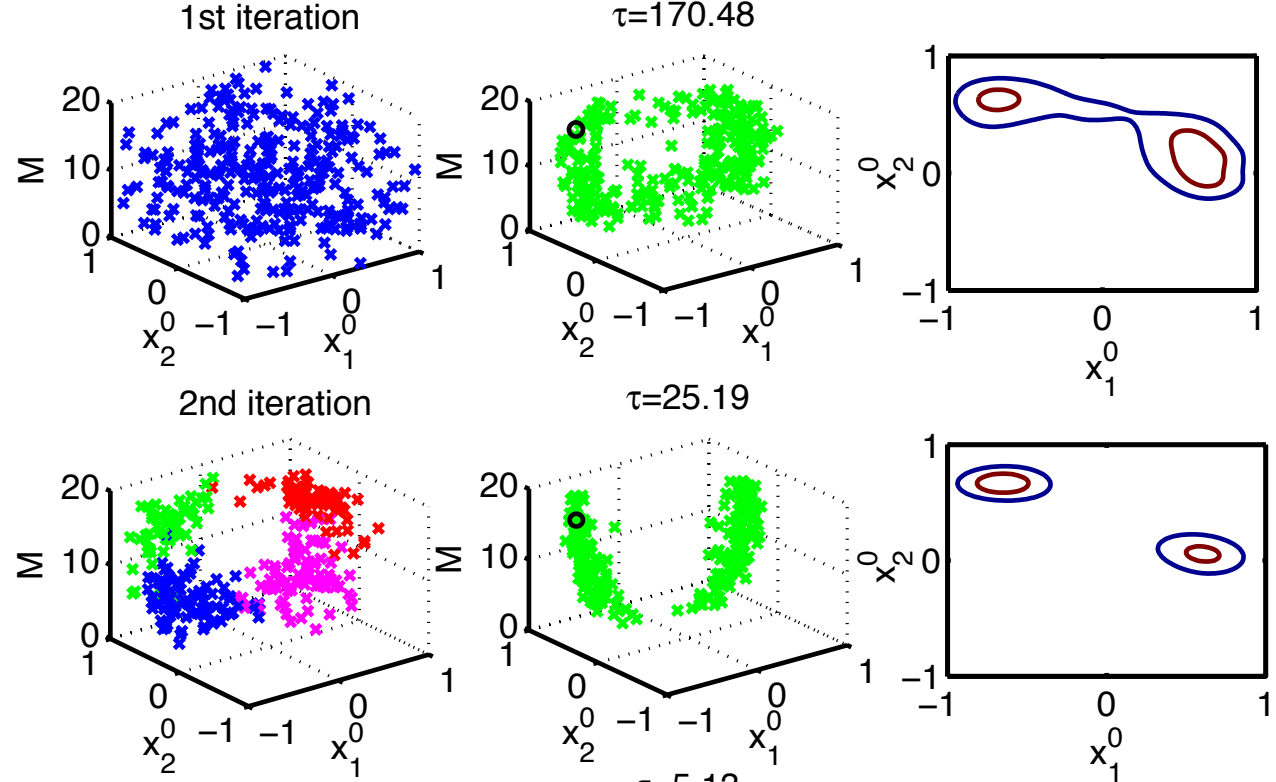

3rd iteration


4th iteration
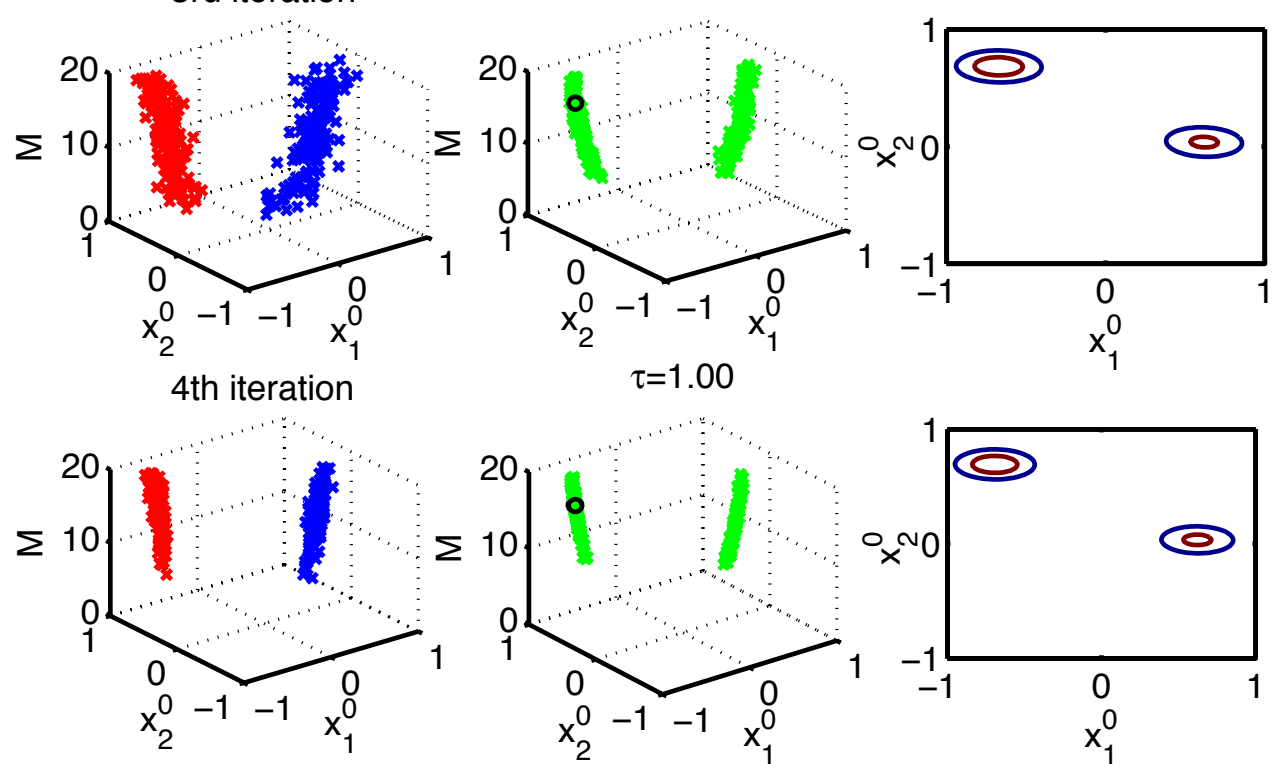

Fig. 4. Adaptive sampling from the posterior pdf (Case 1, Example 1). Left column: proposed points from GM models in each iteration. Different colors represent points from different Gaussian components. Middle column: sample points obtained in each iteration under different temperatures; black circle indicates the reference point. Right column: marginal posterior pdf of source location obtained in each iteration. 

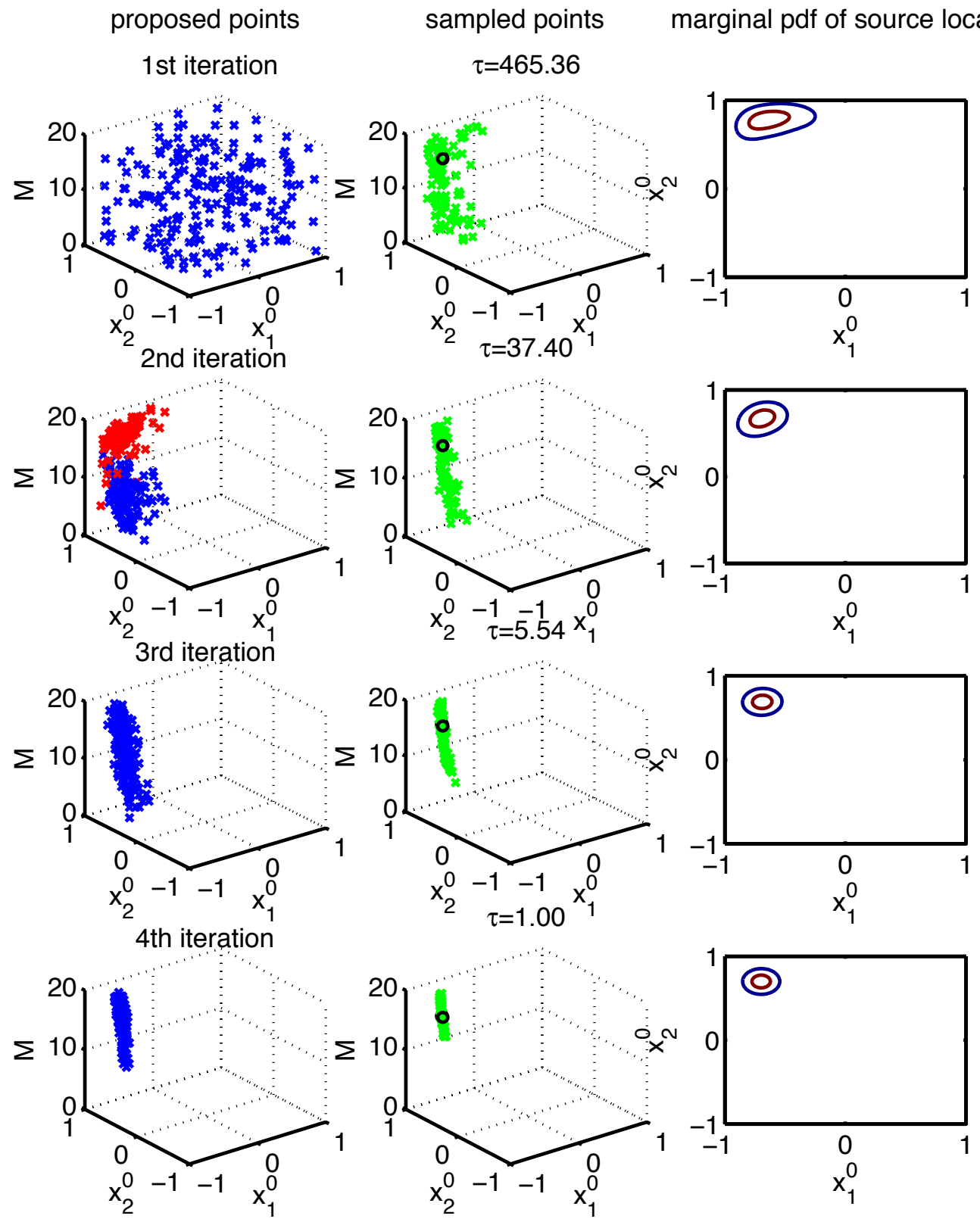

Fig. 5. Adaptive sampling from the posterior pdf (Case 2, Example 1). Left column: proposed points from GM models in each iteration. Different colors represent points from different Gaussian components; black circle indicates the reference point. Middle column: sample points obtained in each iteration under different temperatures. Right column: marginal posterior pdf of source location obtained in each iteration. 


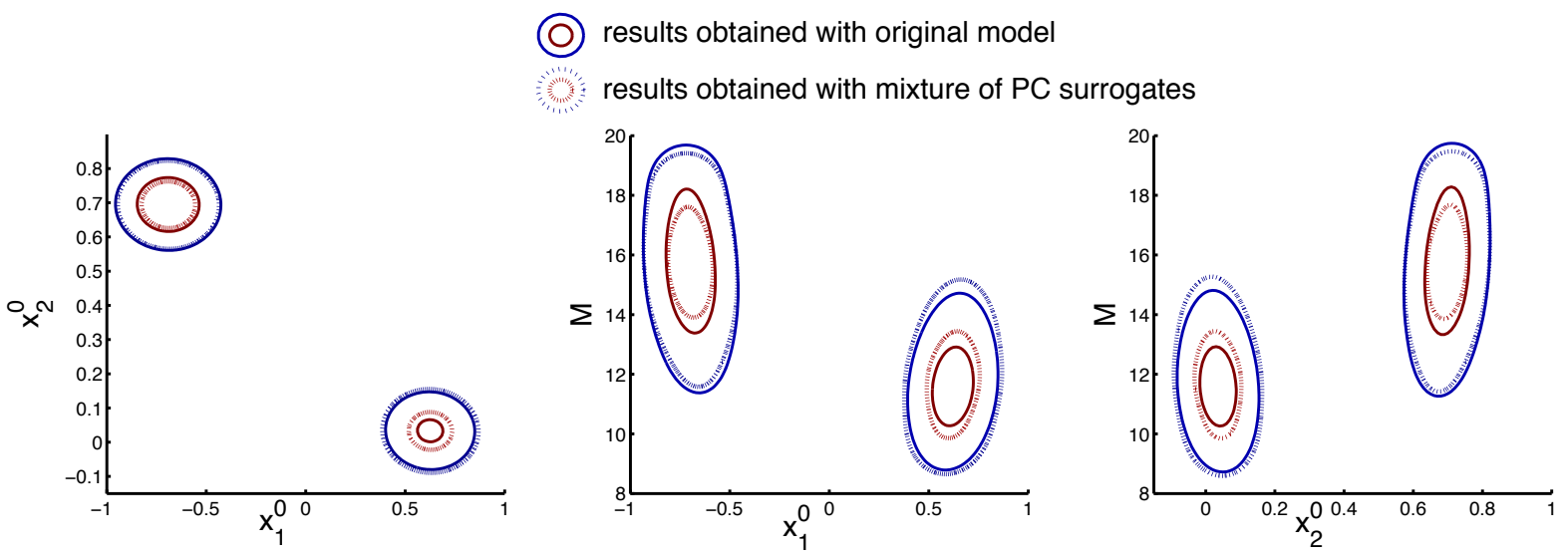

Fig. 6. Comparison between the 2-d marginal pdfs computed from the posterior samples obtained with the original and mixture of PC surrogate models, Case 1, Example 1.

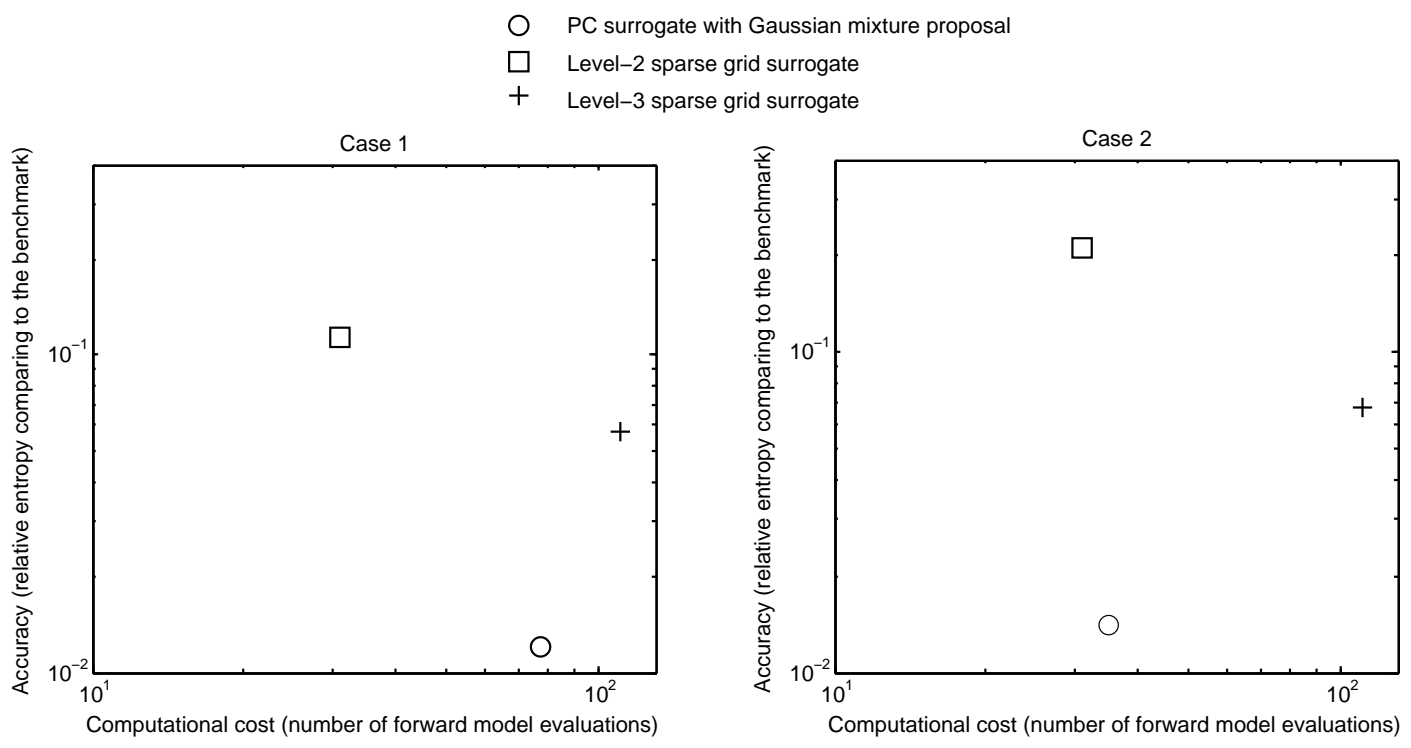

Fig. 7. Accuracy and computation cost comparisons among samples obtained with different surrogates. 


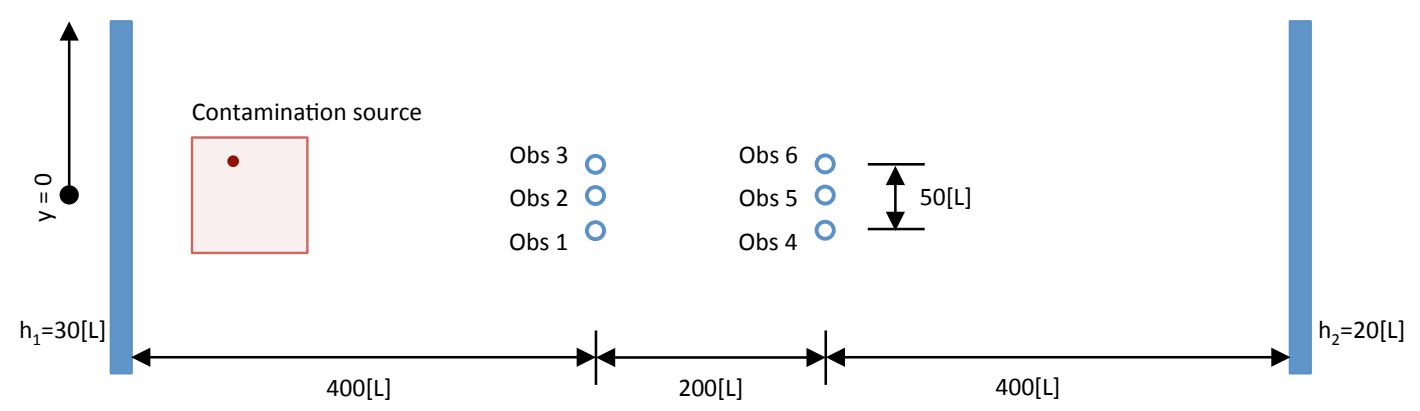

Fig. 8. Schematic representation of the solute transport model (Example 2) [31].
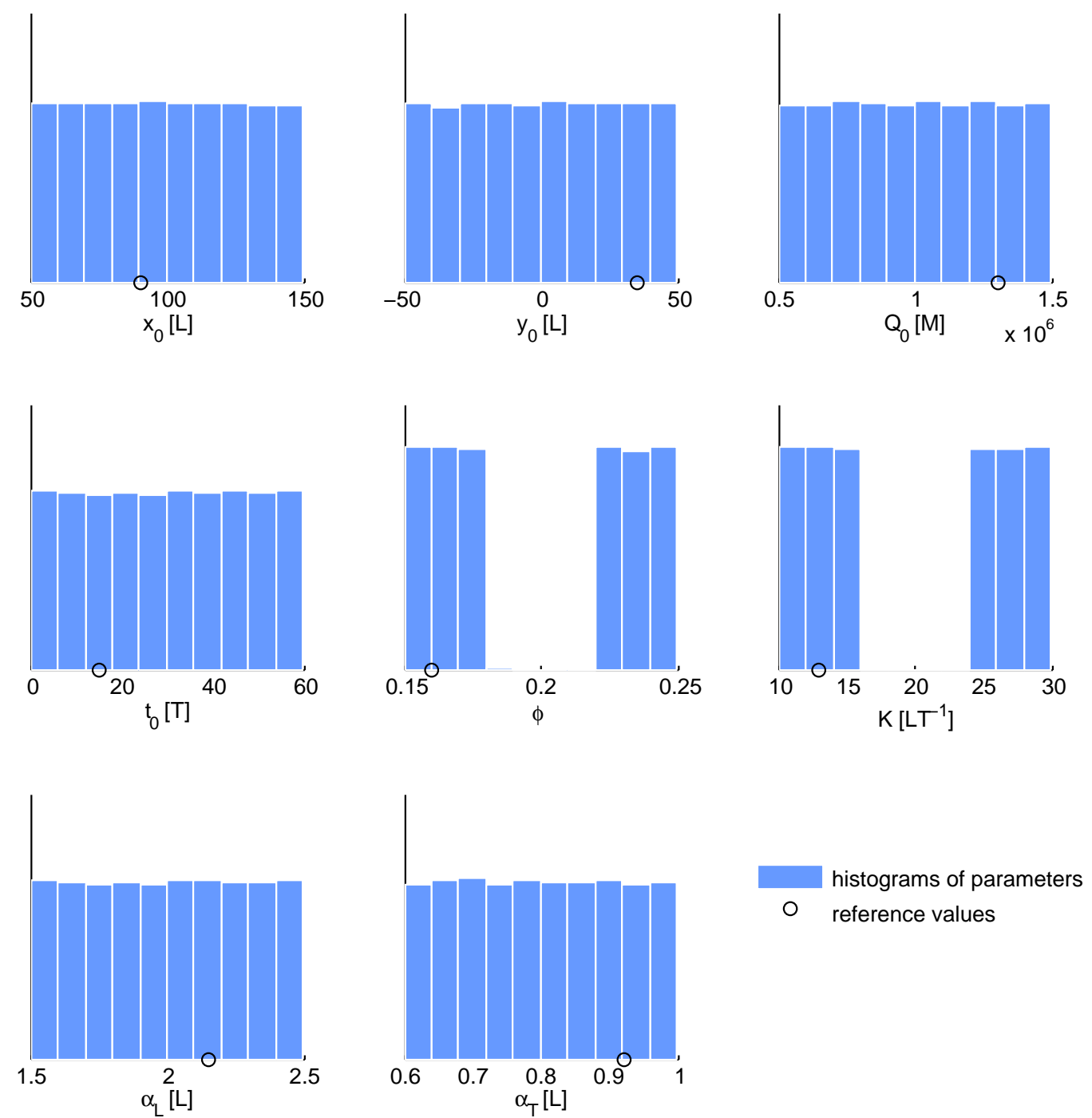

Fig. 9. Prior histograms of each uncertain parameters (Example 2). 

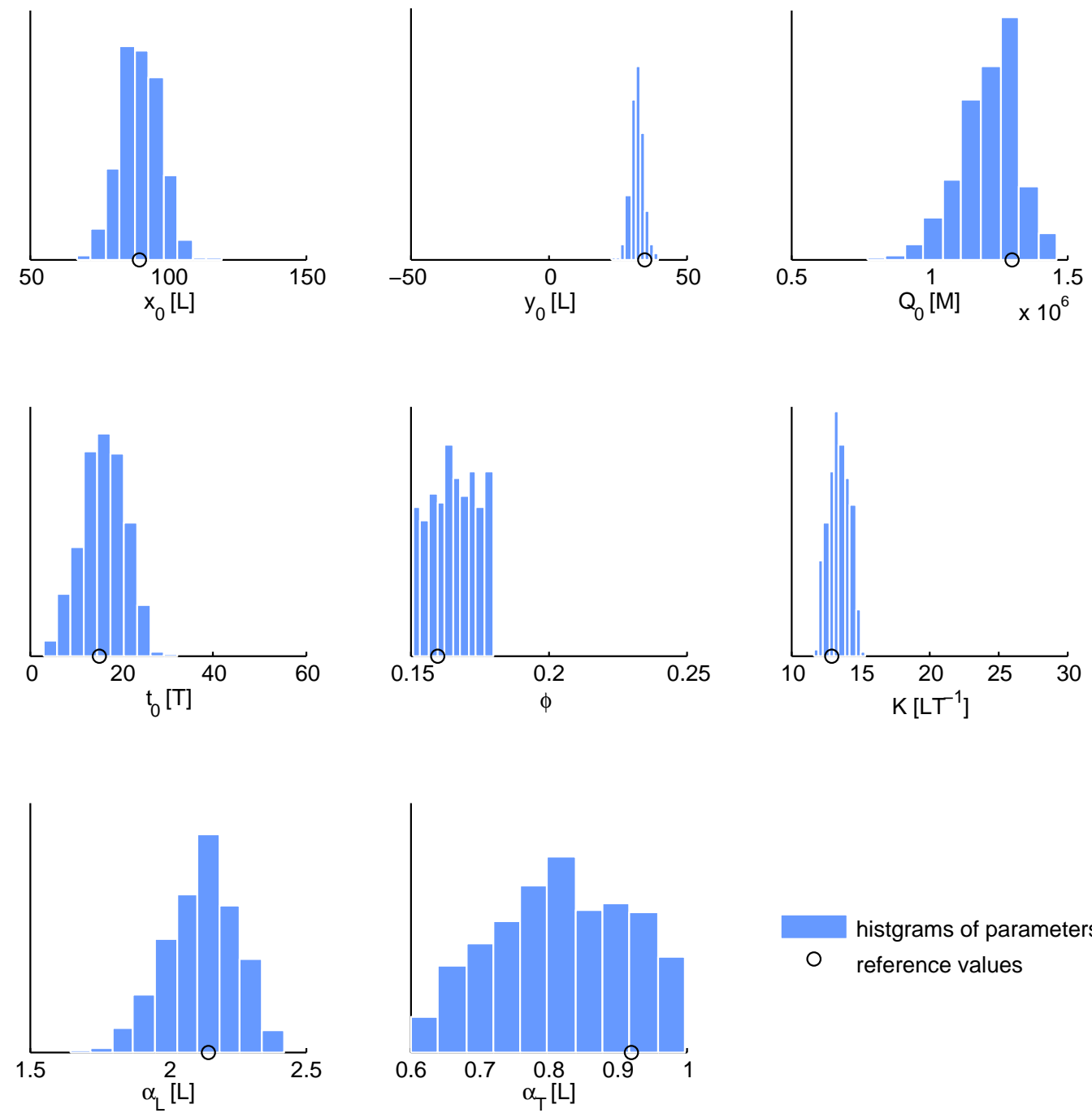

histgrams of parameters

o reference values

Fig. 10. Posterior histograms of each uncertain parameters (Example 2). 


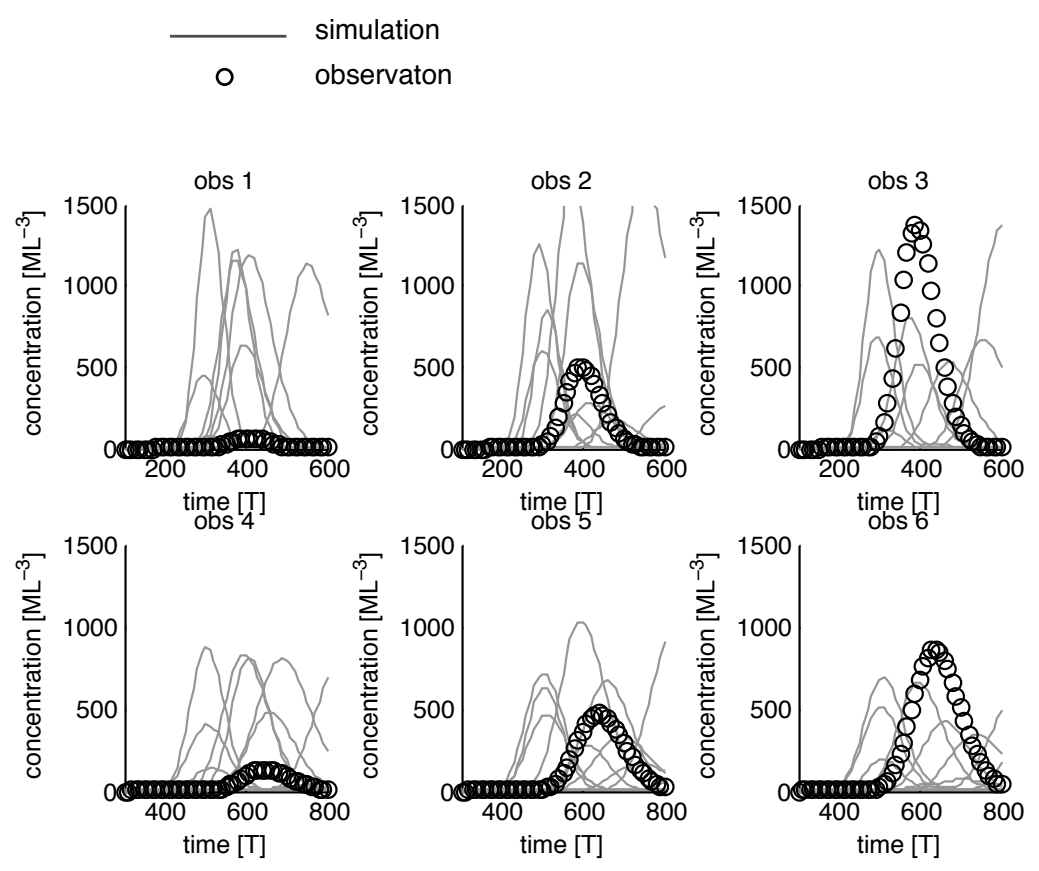

Fig. 11. Concentration breakthrough at observation wells, simulations from prior sample, comparing with observations (Example 2). 


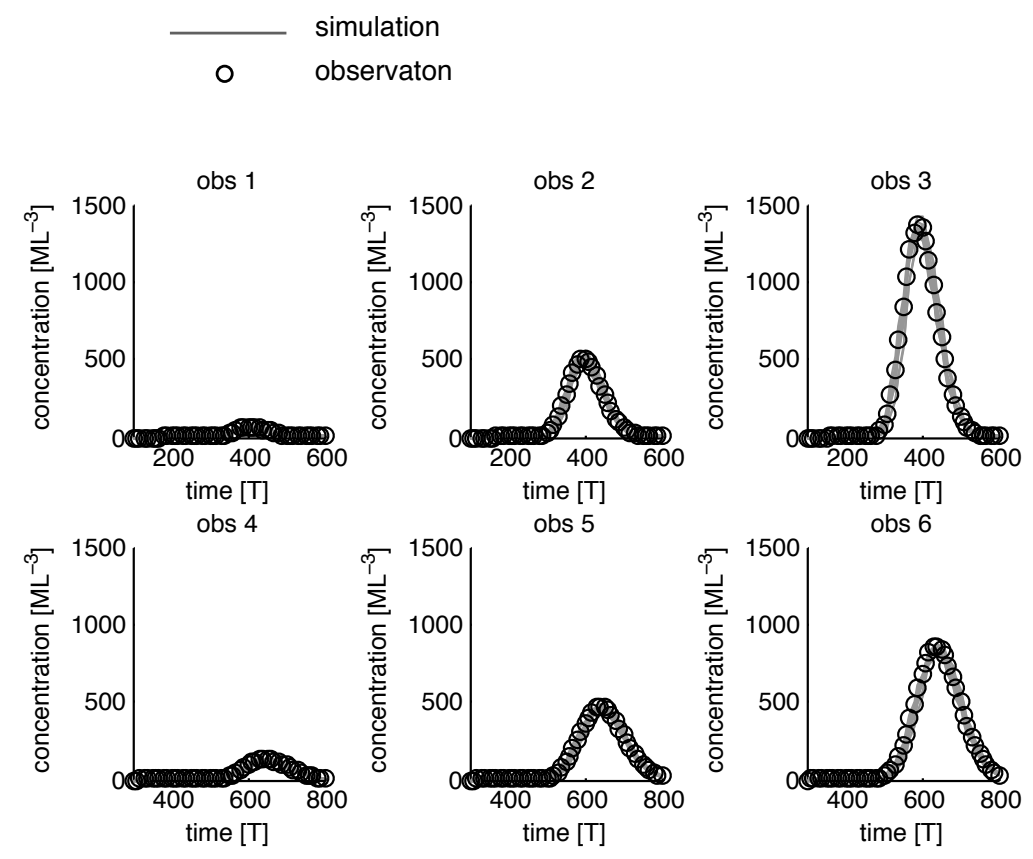

Fig. 12. Concentration breakthrough at observation wells, simulations from posterior sample, comparing with observations (Example 2).



Fig. 13. I-C Fault model [33], distances are measured in feet (Example 3). 
proposed points
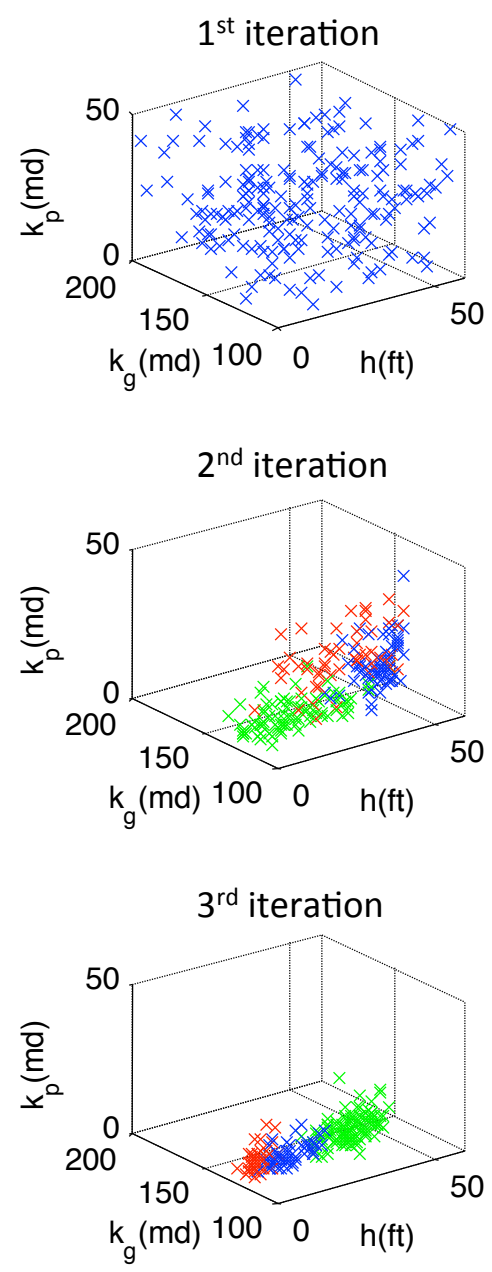

sampled points
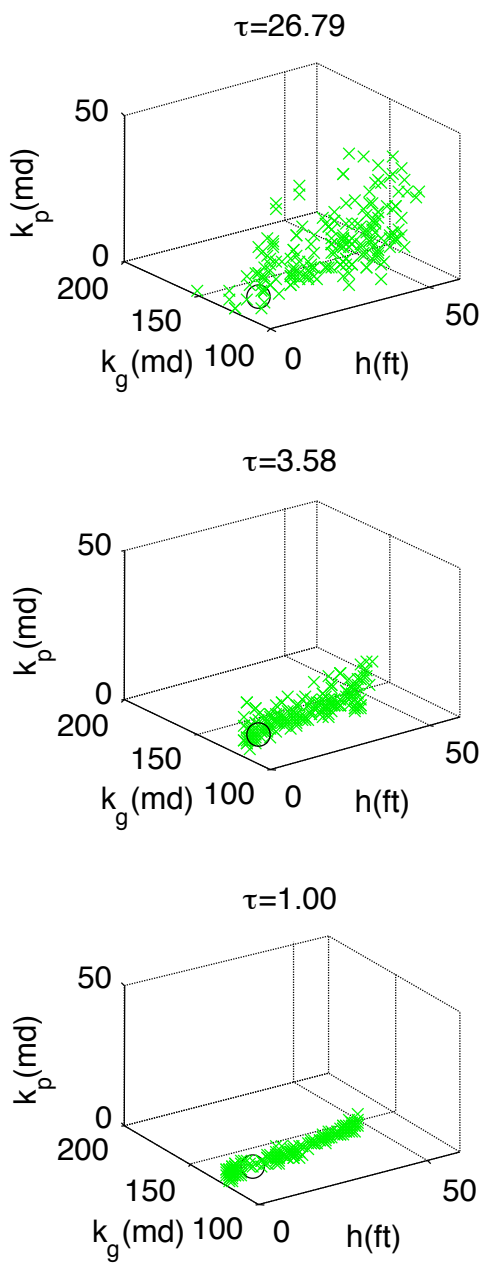

marginal posterior pdf
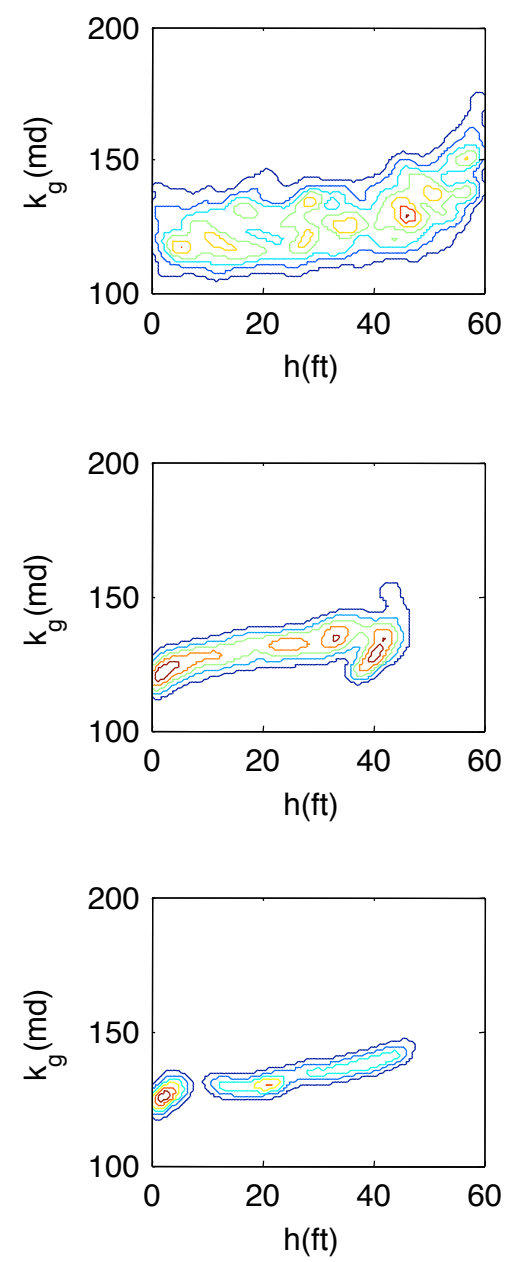

Fig. 14. Adaptive sampling from the posterior pdf (Example 3). Left column: proposed points from GM models in each iteration. Different colors represent points from different Gaussian components. Middle column: sample points obtained in each iteration under different temperatures. Right column: marginal posterior pdf of $k_{g}$ and $h$ in each iteration. 

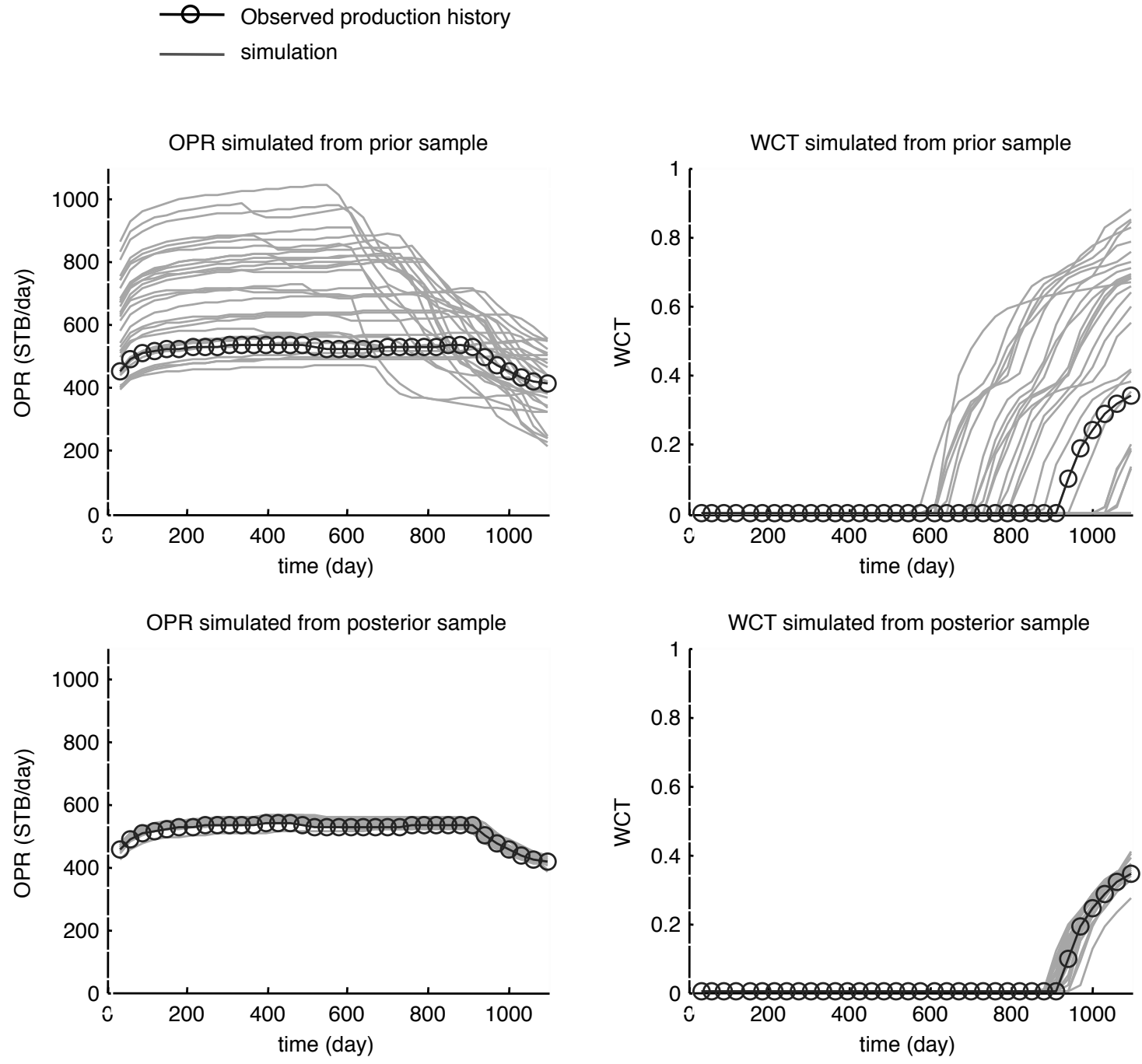

Fig. 15. Simulation results of the I-C Fault model based on the parameter points sampled from prior and posterior distributions (Example 3). 